\title{
Maternal Supply of Cysteamine during Late Gestation Alleviates Oxidative Stress and Enhances Angiogenesis in Porcine Placenta
}

\section{Shuangbo Huang}

South China Agricultural University College of Animal Science

\section{Zifang Wu}

South China Agricultural University College of Animal Science

\section{Xiangyu Hao}

South China Agricultural University College of Animal Science

\section{Zihao Huang}

South China Agricultural University College of Animal Science

\section{Longmiao Zhang}

South China Agricultural University College of Animal Science

Chengjun $\mathrm{Hu}$

South China Agricultural University College of Animal Science

Jianfu Wei

guangzhou dabeinong agri-animal huabandry science and technology Co., Ltd.

Jinping Deng

South China Agricultural University College of Animal Science

chengquan Tan ( $\nabla$ tanchengquan@scau.edu.cn )

South China Agricultural University College of Animal Science https://orcid.org/0000-0003-1686-3778

\section{Research Article}

Keywords: Angiogenesis, Cysteamine, Oxidative stress, Placenta, Sow

Posted Date: February 19th, 2021

DOI: https://doi.org/10.21203/rs.3.rs-211894/v1

License: (1) (i) This work is licensed under a Creative Commons Attribution 4.0 International License.

Read Full License

Version of Record: A version of this preprint was published at Journal of Animal Science and Biotechnology on August 10th, 2021. See the published version at https://doi.org/10.1186/s40104-021- 
00609-8.

Page $2 / 28$ 


\section{Abstract}

Background: Oxidative stress in placenta is associated with the occurrence of adverse pregnancy outcomes in sow, but there are few satisfactory treatment strategies for these conditions. This study investigated the potential of cysteamine (CS) as an antioxidant protectant for regulating the reproductive performance, redox status, and placental angiogenesis of sows.

Methods: The placental oxidative stress status and vascular density of piglets with different birth weights: $<1.0 \mathrm{~kg}$ (low birth weight, LBW) and $1.4-1.6 \mathrm{~kg}$ (normal birth weight, NBW) were evaluated, followed by allotting 84 sows to four treatments $(n=21)$ and feeding them with a basal diet supplemented with $0,100,300$, or $500 \mathrm{mg} / \mathrm{kg}$ of CS (CON, CS100, CS300, and CS500 diet) from day 85 of gestation to day 21 of lactation, respectively. Placentae, serum and colostrum, and blood samples of sows or piglets were collected, and the characteristics of sows and piglets were recorded. Furthermore, the in vivo results were validated using porcine vascular endothelial cells (PVECs).

Results: The placentae for the LBW piglets had higher oxidative damage and lower vascular density than those for the NBW piglets $(P<0.05)$. Further experiments with sows showed that compared with the CON group, the CS100 group was lower in the stillbirth and invalid rates, and higher in the piglet birth weight and placental efficiency $(P<0.05)$. Meanwhile, the CS100 group also displayed higher glutathione and lower malondialdehyde in both the serum and colostrum of sows $(P<0.05)$. Interestingly, compared to the CON group, the LBW placentae of the CS100 group showed a decrease in oxidative damage, while an increase in vascular density $(P<0.05)$, as well as the mRNA level of vascular endothelial growth factor $\mathrm{A}$ and the immunostaining intensity of platelet endothelial cell adhesion molecule- $1(P<0.05)$. Furthermore, the in vitro experiment indicated that CS pre-treatment could significantly reverse the NADPH oxidase 2ROS-mediated inactivation of signal transducer and activator of transcription-3 (Stat3) signaling pathway induced by $\mathrm{H}_{2} \mathrm{O}_{2}$ inhibition of the proliferation, tube formation, and migration of PVECs $(P<0.05)$.

Conclusions: The results indicated that oxidative stress and impaired angiogenesis might contribute to the occurrence of low-birth-weight piglets during pregnancy, but CS supplementation at $100 \mathrm{mg} / \mathrm{kg}$ during late gestation and lactation of sows could alleviate oxidative stress and enhance angiogenesis in placenta, thereby improving pregnancy outcome. The in vitro data showed that the underlying mechanism for the positive effects of CS may be related to the activation of Stat3.

\section{Introduction}

Pregnancy is an oxidative stress challenge for the mother, especially during the late gestation period [1], when the rapid development of the fetus increases the metabolic burdens on pregnant sows or dams, leading to elevated systemic oxidative stress $[2,3]$. Increasing evidence indicates that maternal oxidative stress is associated with several adverse outcomes, such as gestational diabetes mellitus, proteinuria preeclampsia, postpartum hemorrhage, fetal death, and low birth weight $[4,5]$. Thus, reducing oxidative 
stress is a crucial issue for improving the reproductive efficiency of both humans and mammalian animals, but its underlying mechanisms still remain elusive.

The placenta regulates fetal nutritional and hormonal support, which, to a large degree, has a direct impact on the pregnancy outcome [6]. In particular, placental blood vessels play an important role in maternal-fetal material exchange, suggesting the importance of proper placental vasculature development for fetal growth. Moreover, the placenta is highly sensitive to oxidative stress [7], which can cause vascular dysfunction in the placenta $[8,9]$. Our previous studies have uncovered that increased oxidative stress induced by maternal obesity may decrease the development of placental vasculature essential for fetal growth during pregnancy [10], suggesting the involvement of placental oxidative stress in the development of adverse pregnancy outcomes. Mechanically, an increased amount of reactive oxygen species (ROS) can induce autophagy, dysfunction, and apoptotic death of vascular endothelial cells [9]. The foregoing reports indicate that targeting the placenta could be an attractive strategy for modulating oxidative stress-related pregnancy diseases.

Dietary intake of antioxidant nutrients to improve the endogenous antioxidant defense capacity has been considered a plausible way to prevent oxidative stress $[6,11,12]$. Cysteamine (CS), a precursor of glutathione (GSH), is widely used to protect a series of tissues and organs such as gastrointestinal tract [13], brain [14], and kidney [15] from oxidative stress under adverse conditions. We selected CS based on its multiple beneficial modes of action, i.e., CS can improve ileal mucosal health by regulating the oxidation status and apoptosis in finishing pigs [13], and L-cysteine, one of the downstream metabolites of CS can efficiently reduce the inflammatory response in the maternal-fetal interface and improve the placental efficiency in rats [16]. However, no data are available currently regarding the effect of dietary CS supplementation during gestation and lactation on the reproductive performance and antioxidant status of sows, especially the dose-effect relationship. The purpose of this study was to evaluate the effects of CS on sows' reproductive performance, antioxidant status, and placental angiogenesis through an in vitro-in vivo method.

\section{Materials And Methods}

\section{Animals and experimental design}

This study was conducted in Guangzhou DaBeiNong Agri-animal Huabandry Science and Technology Co., Ltd. A total of 84 Landrace $\times$ York sows (parities 2-5) with similar farrowing time were allocated to four dietary treatment groups with each sow as a replicate in a completely randomized design using body weight at 85 day of gestation as a block ( $n=21$ per treatment). The sows in the control group (CON) received a basal gestation or lactation diet without added CS from 85 day of gestation to 21 day of lactation, while the sows in the CS group were fed a basal diet supplemented with 100,300 , or $500 \mathrm{mg} / \mathrm{kg}$ of CS (CS100, CS300, and CS500 diet). All diets were formulated to meet the National Research Council (NRC, 2012) requirements of nutrient standards for gestational and lactational sows. The ingredients and compositions of the basal diet are shown in Supplementary Table S1. 
Sows were housed in individual stalls and fed twice (07:30 and 17:00) a day with a constant amount of 3 $\mathrm{kg}$ during late gestation. During the entire lactation period of 21 days, the piglets had no access to the sow's feed or to creep feed. Sows and piglets were given free access to water throughout the experiment. On the day of farrowing, sows were offered $2.0 \mathrm{~kg}$ of the lactation diet, followed by increasing the amount to $3.0 \mathrm{~kg}$ daily until ad libitum feeding. The reasons for the sows eliminated from this study were recorded in detail and shown in Supplementary Table S2.

\section{Measurements of reproductive performance and sample collection}

After farrowing, the number and weight of the piglets born, born alive, stillbirths and mummies were recorded, and invalid piglets included stillbirth and mummy. Piglets were weaned at 21 day of lactation.

During sow farrowing, umbilical cords were tied with a short silk line and each piglet was marked with a numbered tag to match the individual piglets with their placentae. After placental expulsion and weight recording, the placentae were collected and snap-frozen in liquid nitrogen ( 3 to $4 \mathrm{~cm}$ from the cord insertion point), and the other fresh placental tissues were immediately fixed in $4 \%$ paraformaldehyde. Placental efficiency was calculated by dividing piglet weight by placental weight [17]. In this study, the mean birth weight of the 1181 piglets was $1.4 \pm 0.22 \mathrm{~kg}$ (mean \pm standard error). The placentae were assigned to two categories according to piglet birth weight: $<1.0 \mathrm{~kg}$ (low birth weight, LBW) and 1.4-1.6 kg (normal birth weight, NBW). Litter size at birth was also categorized into 2 classes based on the average number of piglets born alive per litter (15.0 piglets): low yield sow (the number of born alive piglets < 15.0 ) and high yield sow (the number of born alive piglets $\geq 15.0$ ).

Sow blood samples ( $\mathrm{n}=8$ per group) were collected from the ear vein of the fasted gilts using $10 \mathrm{~mL}$ centrifuge tubes at farrowing and at weaning and centrifuged at $3,000 \times \mathrm{g}$ and $4^{\circ} \mathrm{C}$ for $15 \mathrm{~min}$ to recover the serum. The blood samples of piglets were collected from the anterior vena cava of the piglet whose body weight was closest to the average body weight of the litter at birth (NBW piglets, $n=6$ ) on the parturition day and centrifuged at $3,000 \times \mathrm{g}$ and $4^{\circ} \mathrm{C}$ for $15 \mathrm{~min}$ to recover the serum. At $0.5 \mathrm{~h}$ before the birth of the first piglet, colostrum $(20 \mathrm{~mL})$ was collected from the functional glands of each sow. Finally, these samples were stored at $-80{ }^{\circ} \mathrm{C}$ until further analysis of the oxidative parameters.

\section{Oxidative stress parameters in serum, colostrum and placenta}

The total antioxidant capacity (T-AOC), glutathione (GSH), and malondialdehyde (MDA) were determined using the commercial kits (Nanjing Jiancheng Bioengineering Institute, Nanjing, China) according to the manufacturer's procedures. 
Total protein concentrations in placenta and colostrum were measured according to the instructions of the bicinchoninic acid protein assay kit (Beyotime, Beijing, China). T-AOC, GSH, and MDA in placenta and colostrum were normalized to the total protein. T-AOC is associated with the elimination of free radicals and ROS, blocking peroxidation and thus preventing lipid peroxidation and removing catalytic metal ions, while MDA is the end product of lipid peroxidation and an excellent indicator of oxidative stress [18].

\section{Placental vascular density}

Placental tissues fixed in $4 \%$ paraformaldehyde were embedded in paraffin and sectioned at $5 \mu \mathrm{m}$ thickness, followed by staining with hematoxylin and eosin (H\&E). The placental vessels in these areas

were also traced using a projecting microscope (Olympus CX41, Japan) and then quantified as previously described [10].

\section{Cell culture and treatments}

Porcine vascular endothelial cells (PVECs) were obtained from the Cell Bank of the Chinese Academy of Sciences (Shanghai, China) and cultured in 1640 medium with 10\% fetal bovine serum (FPS), $100 \mathrm{U} / \mathrm{mL}$ penicillin, and $100 \mu \mathrm{g} / \mathrm{mL}$ streptomycin at $37^{\circ} \mathrm{C}$ in $5 \% \mathrm{CO}_{2}$ atmosphere. The PVECs with cobblestone morphology were passaged at $90 \%$ confluence and used for experiments within five passages.

Subsequently, the cells were seeded at varying densities incubated for $24 \mathrm{~h}$, and then treated with CS or $\mathrm{H}_{2} \mathrm{O}_{2}$ for the desired time periods. For some experiments, cells were pretreated with various concentrations of CS $(0.5,1$ or $2 \mathrm{mM})$ for $2 \mathrm{~h}$ and/or then treated with various concentrations of $\mathrm{H}_{2} \mathrm{O}_{2}$ $(100,200$ or $300 \mu \mathrm{M})$ for $24 \mathrm{~h}$.

\section{Cell viability assay}

The PVECls (10,000 cells per well) were seeded in 96-well plates and incubated for $24 \mathrm{~h}$. After different treatments, cell viability was measured using cell counting kit-8 assay (CCK-8) (Beyotime) as instructed by the manufacturer. The absorbance of each well at $490 \mathrm{~nm}$ was measured using a microplate reader (Bio-Rad Laboratories, Hercules, CA).

\section{Measurement of intracellular ROS generation}

The accumulation of intracellular ROS was examined using the ROS assay kit (Beyotime) according to the manufacturer's instructions. Briefly, cells were grown in a 96-well plate and subjected to different treatments. After incubation with $10 \mu \mathrm{M}$ 2,7-dichlorofluorescein diacetate at $37^{\circ} \mathrm{C}$ for $20 \mathrm{~min}$, the fluorescence intensity of the the cells was measured using the fluorescence plate reader (BD Falcon, Bedford, MA, USA) at Ex./Em. $=488 / 525 \mathrm{~nm}$. 


\section{Scratch healing assay}

The wound healing scratch assay was used to assess cell migration as previously described [19]. Briefly, cells were seeded onto a 6-well plate and cultured overnight until the formation of a confluent monolayer, followed by making a scratch wound with a 200- $\mu \mathrm{L}$ pipette tip and measuring the effects of $\mathrm{H}_{2} \mathrm{O}_{2}, \mathrm{CS}$, and stattic on scratch healing at $24 \mathrm{~h}$ after the scratch. The images of the wounded areas were captured using an Olympus inverted microscope and quantified using the ImageJ software.

\section{In vitro tube formation assay}

Matrigel tube formation assays were used to assess the in vitro angiogenic activity of PVECs. Briefly, after experimental treatment, PVECs were seeded in 96-well plates precoated with $50 \mu \mathrm{L}$ Matrigel (BD company, USA) at a density of $1 \times 10^{4}$ cells per well. After $4 \mathrm{~h}$ incubation, Matrigel-induced morphological changes in HRGECs and their tubular networks were photographed at 50 or $100 \times$ magnification for analysis using Image $\mathrm{J}$ software.

\section{Trans-well migrationassay}

The chemotactic migration of PVECs was assayed using a trans-well chamber equipped with a polycarbonate filter with a diameter of $6.5 \mathrm{~mm}$ and a pore size of $8 \mu \mathrm{m}$. Briefly, after different treaments, PVECs were suspended in 1640 medium to a final concentration of $5 \times 10^{4}$ cells $/ \mathrm{ml}$ and were placed in the upper wells of the chamber, while the lower chamber was filled with $600 \mu$ medium containing $10 \%$ FPS. After incubation at $37^{\circ} \mathrm{C}$ for $48 \mathrm{~h}$, the cell culture inserts were collected. The cells on the upper side of the filters were removed with cotton-tipped swabs, while the cells on the underside of the filters were fixed with $4 \%$ formaldehyde for 30 min, which were stained with crystal violet for 20 min and counted in five randomly chosen fields.

\section{Quantitative real-time RT-PCR (qRT-PCR) analysis}

Total RNA from placenta or PVECs was extracted with the reagent box of Total RNA Kit according to the manufacturer's instructions. The concentration of RNA was quantified using a NanoDrop ${ }^{\circledR} 2000$ (Thermo Fisher, USA). After reverse transcription using Primer Script TM RT reagent Kit (Takara, Qingdao, China), quantitative PCR (qPCR) was conducted using SYBR Green on a QuantStudio 6 RealTime PCR System (Thermo Fisher, USA) under the conditions of denaturation at $95^{\circ} \mathrm{C}$ for $10 \mathrm{~min}$, amplification at $95{ }^{\circ} \mathrm{C}$ for $15 \mathrm{~s}$ and $60^{\circ} \mathrm{C}$ for $1 \mathrm{~min}$ for 40 cycles. Each target gene was individually normalized to the reference gene $\beta$-actin by using the quantification method of $2^{-\Delta \Delta c t}$. Primers used in this study are shown in Supplemental Table S3. 


\section{Western blotting}

Total proteins were extracted from PVECs using the protein extraction kit (Beyotime, Beijing, China) according to the manufacturer's guide. Briefly, an amount of $10 \mu \mathrm{g}$ protein was loaded and separated by SDS-PAGE gel electrophoresis, followed by transferring the proteins onto the polyvinylidenedi fluoride membranes (Merck Millipore). After blocking with TBS/T buffer containing 5\% milk, the membranes were incubated with the primary antibodies against vascular endothelial growth factor A (VEGF-A) (19003-1-AP, Proteintech, USA, 1:1000), NADPH oxidase 2 (NOX2) (19013-1-AP, Proteintech, USA, 1:1000), signal transducer and activator of transcription-3 (Stat3)(ab76315, Abcam, USA, 1:1500), p-Stat3 (ab68153, Abcam, USA, 1:1500), and $\beta$-actin (4970, CST, USA, 1:1000). Subsequently, the membranes were incubated with appropriate HRP-conjugated anti-rabbit IgG secondary antibody (AS014, Abclonal, China, 1:5000). Images were captured using the ChemiDoc MP system (Bio-Rad, Hercules, CA, USA), and band densities were quantified using Image Lab software (Bio-Rad, Hercules, CA, USA) and then normalized to $\beta$-actin content.

\section{Immunofluorescence}

Placental tissues fixed in $4 \%$ paraformaldehyde were embedded in paraffin and sectioned at $5 \mu \mathrm{m}$ thickness for platelet endothelial cell adhesion molecule-1 (CD31) immunofluorescence as described previously [19].

\section{Statistical analysis}

Data are presented as mean \pm SEM and were statistically analyzed using one-way ANOVA and Duncan's multiple-range test in SPSS 20.0 (SPPS Inc., Chicago). Tamhane's T2 test was used to assess variance heterogeneity. The stillbirth, LBW, and invalid rates were analyzed using the Chi-square test. Pearson's correlation coefficient was used to analyze the correlation between piglet birth weight and placental vascular density, T-AOC, and MDA. Additionally, polynomial contrasts were used to evaluate the linear and quadratic effects of CS supplementation on the various parameters measured in the sow experiment. Differences were considered significant at $P<0.05$, and a tendency was considered at $0.05 \leq P<0.1$.

\section{Results}

\section{Correlation between birth weight and placental characteristics of piglets}

When compared with the NBW placenta, the LBW placenta showed a decrease $(P<0.05)$ in both the blood vessel density (Figure 1A, B) and the immunostaining intensity of CD31 (biomarker of the endothelial cell in small vessels) (Figure 1C, D). Meanwhile, piglet birth weight showed a significant 
positive correlation with placental vascular density $\left(R^{2}=0.7146, P<0.0001\right)$ (Figure 1E), or T-AOC in placenta $\left(R^{2}=0.2744, P=0.0086\right)($ Figure $1 F)$, while a negative correlation with placental MDA level $\left(R^{2}=\right.$ $0.3700, P=0.0016)$ (Figure 1G).

\section{Reproductive performance}

The four groups showed significant differences in sow performance (Figure 2). When compared with CON group, CS100 group was significantly lower $(P<0.05)$ in stillbirth and invalid piglet rates, while higher in the piglet birth weight $(P<0.05)$, and the CS500 group had a lower proportion of LBW piglets $(P<0.05)$ in low yield sows.

\section{Oxidative stress parameters in serum, colostrum and placenta}

As shown in Figure 3A-C, compared with the CON group, the sows in the CS500 group showed an increase in the serum T-AOC levels at farrowing and weaning and an increase of serum GSH levels at farrowing $(P<0.05)$, and the sows in the CS100 group showed an increase of serum GSH leves at farrowing and weaning $(P<0.05)$. For these parameters in colostrum, when compared with the CON group, the CS100 group had a higher colostrum GSH level but a lower colostrum MDA level $(P<0.05)$ (Figure 3D-F). Additionally, these oxidative stress parameters in neonatal serum were also investigated ( $P$ $<0.05$ ) (Figure 3G-I), and the serum GSH level of NBW piglets was significantly higher in the CS500 group than in the CON group $(P<0.05)$.

Importantly, we observed that the placentae of piglets with different birth weights react differently with maternal CS supply (Figure 4). Compared with the CON group, the CS100 group showed higher contents of T-AOC $(P<0.05)$ and GSH $(P<0.05)$ in the LBW placenta, with no difference observed in the NBW placentae among the four dietary treatments.

The effects of CS on placental function were further explored by analyzing the placental vascular density and the expression of angiogenesis-related genes. Figure $5 \mathrm{~A}, \mathrm{~B}$ presents the effects of CS supplementation on the placental vascular density in LBW or NBW piglets. The placental vascular density showed an increase in the LBW placenta of the CS100 group versus the CON group $(P<0.05)$. For NBW placenta, the CS supplementation did not significantly change the placental vascular density. Immunostaining analysis also revealed higher expression levels of CD31 in the LBW placenta in the CS100 group versus the CON group $(P<0.05)$ (Figure $5 C, D)$, and similar results were also found in the mRNA level of VEGF-A $(P<0.05)$ (Figure $5 \mathrm{E})$.

Collectively, CS has positive effects on antioxidant capacity and placental angiogenesis of sows. 


\section{Oxidative stress induced by $\mathrm{H}_{2} \mathrm{O}_{2}$ inhibits tube formation and migration in vitro.}

The relationship between placental oxidative status and angiogenesis was further investigated by using PVECs to evaluate the effects of oxidative stress on angiogenesis in vitro. As shown in Figure 6A-B, compared with the control group, the viability of PVECs was significantly decreased $(P<0.05)$ under $\mathrm{H}_{2} \mathrm{O}_{2}$ treatment in a dose- and time-dependent manner. Whether the redox state of PVECs can be changed by $\mathrm{H}_{2} \mathrm{O}_{2}$ was investigated by analyzing the mRNA expression of antioxidant-related genes and the endoplasmic stress markers. The mRNA expression level showed a significant decrease $(P<0.05)$ for GPX1, SOD1, SOD2 and CAT while a significant increase for $G R P 78(P<0.05)$ in a dose-dependent manner (Figure 6C). The ROS level also increased significantly $(P<0.05)$ in a dose-dependent manner (Figure 6D, E). Meanwhile, tube formation (Figure 6F, G) and migration (Figure 6H, I) were significantly impaired $(P<0.05)$ in PVECs after treatment with $\mathrm{H}_{2} \mathrm{O}_{2}$ for $24 \mathrm{~h}$. As $200 \mu \mathrm{M} \mathrm{H}_{2} \mathrm{O}_{2}$ treatment for $24 \mathrm{~h}$ could significantly decrease the viability, tube formation and migration to about $50 \%$ and change the redox state of PVECs, this concentration was used for further experiments.

\section{Oxidative stress impairs the Stat3/VEGF-A pathway in PVECs}

A growing body of evidence supports an important role of Stat3 in placental angiogenesis [20]. In our previous study, Stat3/VEGF-A pathway was found to be impaired in LBW placenta (Hu et al., unpbulished data), leading to the question of whether $\mathrm{H}_{2} \mathrm{O}_{2}$-mediated angiogenesis inhibition is controlled by Stat3. After treatment with $\mathrm{H}_{2} \mathrm{O}_{2}$ for 24, the relative mRNA expression of Stat3-regulated genes (IL6, IL8, and VEGF-A) in PVECs significantly decreased $(P<0.05)$, in contrast to a signifcant increase $(P<0.05)$ in the expression of NOX2 (the upstream negative regulator of Stat3) (Figure 7A-B). Similar results were obtained from Western blotting analysis (Figure 7C, D).

\section{CS rescues $\mathrm{H}_{2} \mathrm{O}_{2}$-induced Stat3 signaling pathway activity}

The effects of CS on ROS level and endothelial dysfunction in $\mathrm{H}_{2} \mathrm{O}_{2}$-induced PVECs were also evaluated. As shown in Figure 8A, E, the ROS level induced by $\mathrm{H}_{2} \mathrm{O}_{2}$ was reduced in the cells pretreated with CS $(P<$ 0.05 ). In addition, the impaired migration by scratch healing assay $(P<0.05)$ (Figure $8 \mathrm{~B}, \mathrm{~F}$ ) or by transwell assy $(P<0.05)$ (Figure 8C, G), and tube formation $(P<0.05)$ (Figure 8D, $\mathrm{H}$ ) induced by $\mathrm{H}_{2} \mathrm{O}_{2}$ were partially recovered in PVECs pretreated with CS $(P<0.05)$. Similarly, $\mathrm{H}_{2} \mathrm{O}_{2}$ resulted in a significant decrease in cell viability, but CS supplementation can alleviate the negative effect of $\mathrm{H}_{2} \mathrm{O}_{2}$ on cell viability $(P<0.05)$ (Figure 81). 
We hypothesized that Stat3/VEGF-A might mediate the enhanced endothelial function in oxidative stress with CS supplementation. Importantly, $\mathrm{CS}$ was seen to largely reverse the $\mathrm{H}_{2} \mathrm{O}_{2}$-downregulated Stat3/VEGF-A activity $(P<0.05)$ (Figure 9A, B). Furthermore, pretreatment with stattic, a selective Stat3 inhibitor, abrogated the CS-mediated pro-angiogenesis $(P<0.05)$ (Figure 9C, D) and pro-viability $(P<0.05)$ (Figure 9E) effects against $\mathrm{H}_{2} \mathrm{O}_{2}$ in PVECs. Meanwhile, the inhibition of Stat3 decreased the cell viability and induced a significance decrease in the VEGF-A protein level in CS pretreatment with $\mathrm{H}_{2} \mathrm{O}_{2}$-cultured PVECs $(P<0.05)$ (Figure 9F, G).

The above data confirmed that the Stat3/VEGF-A signaling pathway is invovled in the endothelial protection of CS against oxidative stress.

\section{Discussion}

Previous studies have shown that increased oxidative stress in placenta is associated with the occurrence of adverse pregnancy outcomes, including intrauterine growth restriction and stillbirth [21, 22], suggesting that oxidative stress may contribute to the occurrence of low birth weight and stillbirth. However, the molecular mechanism underlying this link remains unclear. In pigs, high occurrence of intrauterine growth restriction and stillbirth is widely reported [23]. Additionally, pigs are one of the most used animals in biomedical studies on human pregnancy, primarily because of their physiological similarities to humans $[24,25]$. The objective of this study was to investigate whether maternal CS supplementation during late gestation improves pregnancy outcomes by examining the changes in antioxidant status and placental angiogenesis. Our study demonstrated that the placentae for low birth weight neonates were vulnerable to oxidative stress, which might contribute to the occurrence of low birth weight piglets during pregnancy. Additionally, CS was shown to have great potential to improve pregnancy outcomes in sows, including birth weight and stillbirth, by reducing oxidative stress and enhancing angiogenesis in placenta.

Placenta plays an important role in fetal growth [26]. Thus, differences in the placental oxidation parameters of piglets with different birth weights were first determined in the present study. We found that birth weight was positively correlated with T-AOC, but negatively correlated with MDA, which was in line with Takagi et al., who reported that placental oxidative DNA damage was increased in intrauterine growth restriction [7]. Excessive ROS can be produced at certain windows in placental development and in some pathological pregnancies, such as those complicated by preeclampsia and/or intrauterine growth restriction, overpowering antioxidant defenses with deleterious outcome [27, 28]. Placental blood vessels are important for fetal growth and development [29], and increased oxidative stress may have a significant effect on placental function, including the proliferation and differentiation of trophoblast cells and vascular reactivity $[8,30]$. Likewise, in this study, the placentae for LBW piglets showed increased oxidative damage and was vulnerable to angiogenesis impairment. Particularly, oxidative stress induced by $\mathrm{H}_{2} \mathrm{O}_{2}$ (a major type of ROS) prompted intracellular ROS generation and inhibited the tube formation and migration of PVECs as well as the expression of VEGF-A (a major driver for blood vessel formation) 
in vitro. These findings suggest that birth weight is associated with placental redox state, angiogenesis, or both.

Increased oxidative stress during late gestation could lead to a greater percentage of stillbirth [31, 32], which is a substantial cause of economic loss in livestock [33]. Our previous data indicated that alleviating serum oxidative stress at day 109 of gestation contributed to reducing the stillbirth of sows [18]. In this study, CS supply during late gestation was shown to improve the pregnancy outcomes, including birth weight and stillbirth in sows. To test whether the mechanism of CS in improving pregnancy outcomes is related to oxidative stress, we analyzed the effect of dietary CS supplementation on the related parameters in serum and colostrum, and found dietary CS supplementation could partially improve the antioxidant status of sows and their offspring as well as reduce the oxidative stress parameters. Meanwhile, maternal CS supplementation was also shown to partially increase the antioxidant status of placenta for LBW piglets. CS is a known antioxidant and anti-inflammatory agent [34], and previous studies have proposed a number of mechanisms for its protection against oxidative stress. Firstly, CS was reported as a contributor to the cellular redox state of hepatocytes by acting through sulfhydryl-disulfide exchange reactions in cells [34]. Secondly, CS can inhibit nucleotide-binding oligomerization domain-like receptor containing pyrin domain 3 inflammasome by metabolizing cysteine, thereby improving the redox status of maternal-placental interface in sows [11]. Moreover, when used at low concentrations, CS can promote the transport of cysteine into cells, which can be further used to synthesize GSH (one of the most potent intracellular antioxidants) and influence the cellular redox homeostasis [35]. GSH status can reflect the antioxidant capacity of developing embryos. Previous studies have shown that GSH can reduce the formation of ROS and ultimately protect embryos against oxidative stress $[36,37]$. In guinea pig, supplementation with GSH precursor $\mathrm{N}$-acetylcysteine was shown to normalize the endothelial function in intrauterine growth-restricted placenta and fetus, thereby normalizing fetal growth [38]. In the present study, the GSH levels were elevated in the LBW placentae of the CS100 group, which is beneficial to the growth and survival of embryo and protects the fetus against ROS damage.

Another important finding is that the dose-effect relationship between CS supplementation and the redox state and pregnancy outcomes is not linear, and $100 \mathrm{mg} / \mathrm{kg}$ (minimum dose) was shown as the suitable supplemental dose. High doses of CS can diminish the activity of glutathione peroxidase, the enzyme that catalyzes the oxidation of GSH to its disulfide [39], indicating that the divergent results of studies using CS can frequently be explained by the antioxidant effect of the drug, which can be counteracted by its direct toxicity when higher doses are used [35]. However, the potential mechanism needs to be revealed by further studies.

The dense blood vessel network in the placenta is responsible for the exchange of respiratory gases, nutrients, and wastes between mother and fetus throughout pregnancy, which is essential for normal fetal growth [40]. Throughout gestation, the vasculature of the placenta is constantly evolving to accommodate the mounting demands of the fetus and can be directly influenced by a number of exogenous factors, such as maternal diet, smoking, and medication use [41]. Therefore, the effect of CS 
on placental vessel density was evaluated in the present study, and $100 \mathrm{mg} / \mathrm{kg}$ CS dietary supplementation was found to increase the placental vessel density in LBW piglets. CD31 is a biomarker of endothelial cells in blood vessels, and VEGF-A is a major driver of blood vessel formation [19]. The increased CD31 immunofluorescence intensity and VEGF-A mRNA level further demonstrated that the placental vessel density was higher in the CS100 group than in the other three groups. It is worth noting that changes in placental vessel density were consistent with oxidative stress levels only in the LBW placenta among the four groups, further implying that oxidative stress may play an important role in placental angiogenesis. Another key factor for efficient nutrient exchange is placental efficiency [17]. Variations in placental efficiency, a measure of grams of fetus produced per gram of placenta, were initially studied between swine breeds, where increased placental efficiency was associated with larger litters. For instance, when compared to Yorkshire pigs, Meishan pigs known for large litter sizes had smaller placentae, but higher vascularity [42]. These observations seem logical in that proper placental vascularization facilitates the efficient exchange of nutrients, implying the importance of improving overall placental efficiency [43], which may also help explain the increased placental efficiency in CS100 group. Overall, maternal CS supply can decrease the oxidative stress level, thus enhancing angiogenesis in placentae.

Previous studies have reported Stat3 as an important regulator of the adaptive response to oxidative stress in the placenta, which may be related to the increased oxidative stress and the altered trophoblast invasion and placental angiogenesis [41]. The expression of Stat3 and Stat3-mediated genes was shown to be downregulated in our recent study (unpublished data). In the present study, Stat3 targeting genes (VEGF-A, IL-6, and IL-8) and upstream negative regulators (NOX2) were used to validate the in vitro modulation of $\mathrm{H}_{2} \mathrm{O}_{2}$. The data of our study and others have demonstrated that $\mathrm{H}_{2} \mathrm{O}_{2}$ inhibits the proliferation and migration of PVECs by upregulating NOX2-ROS-mediated inactivation of Stat3 signalling pathway in vitro [44], indicating that Stat3 may play an important role in placental angiogenesis in porcine. Previous studies have also shown that CS could activate Stat 3 signaling and provide the critical protection required for the survival of intestinal epithelial cells in vivo [45]. Here, we found a novel function of $\mathrm{CS}$, modulating the repair of $\mathrm{H}_{2} \mathrm{O}_{2}$-induced PVEC oxidative stress through the Stat3 signaling pathway. The pretreatment of PVECs with statttic (a selective Stat3 inhibitor) and CS failed to prevent the decrease of VEGF-A protein expression and tubification ability induced by $\mathrm{H}_{2} \mathrm{O}_{2}$, strongly suggesting that Stat3 mediates the endothelial protective effect of CS in vitro.

\section{Conclusions}

In this study, oxidative stress and impaired angiogenesis were shown to contribute to the occurrence of low-birth-weight piglets during pregnancy, but maternal CS supply during late gestation and lactation of sows could alleviate oxidative stress and enhance angiogenesis in placenta, thereby enhancing pregnancy outcomes, including increased birth weight in low yield sows and reduced stillbirth. The in vitro data showed that the underlying mechanism for the positive effects of CS may be related to Stat3 activation. 


\section{Declarations}

\section{Acknowledgements}

The authors would like to thank the Guangzhou DaBeiNong Agri-animal Huabandry Science and Technology Co., Ltd. with limited liability for animal management.

\section{Funding}

This project was funded by Project of National Natural Science Foundation of China (No. 31872985).

\section{Availability of data and materials}

The datasets produced and/or analyzed during the current study are available from the corresponding author on reasonable request.

\section{Authors' contributions}

The author contributions are as follows: C.Q.Tan, J.P. Deng and S.B. Huang conceived and designed the experimental plan. S.B. Huang, Z.F. Wu, X.Y. Hao and Z.H. Huang were involved in the animal experiments, analysis and data collection. S.B. Huang wrote the manuscript. C.J. Hu, J.F. Wei and L.M. Zhang assisted with the manuscript preparation.

\section{Ethics approval and consent to participate}

All procedures involving animals were approved by the Animal Ethics Committee of South China Agricultural University and followed the Guidelines for the Care and Use of Laboratory Animals of South China Agricultural University (Guangzhou, China).

\section{Consent for publication}

Not applicable.

\section{Competing of interest}

There were no conflicts of interest.

\section{References}


1. Xu M, Che L, Gao K, Wang L, Yang X, Wen X, et al. Effects of Dietary Taurine Supplementation to Gilts during Late Gestation and Lactation on Offspring Growth and Oxidative Stress. Animals (Basel). 2019;9.

2. Tan C, Wei H, Sun H, Ao J, Long G, Jiang S, et al. Effects of Dietary Supplementation of Oregano Essential Oil to Sows on Oxidative Stress Status, Lactation Feed Intake of Sows, and Piglet Performance. Biomed Res Int. 2015;2015:525218.

3. Tan C, Wei H, Ao J, Long G, Peng J. Inclusion of Konjac Flour in the Gestation Diet Changes the Gut Microbiota, Alleviates Oxidative Stress, and Improves Insulin Sensitivity in Sows. Applied and environmental microbiology. 2016;82:5899-909.

4. Pereira AC, Martel F. Oxidative stress in pregnancy and fertility pathologies. Cell Biology and Toxicology. 2014;30:301-12.

5. Kim SW, Weaver AC, Shen YB, Zhao Y. Improving efficiency of sow productivity: nutrition and health. J Anim Sci Biotechnol. 2013;4:26.

6. Meng Q, Guo T, Li G, Sun S, He S, Cheng B, et al. Dietary resveratrol improves antioxidant status of sows and piglets and regulates antioxidant gene expression in placenta by Keap1-Nrf2 pathway and Sirt1. Journal of Animal Science and Biotechnology. 2018;9:639-51.

7. Takagi Y, Nikaido T, Toki T, Kita N, Kanai M, Ashida T, et al. Levels of oxidative stress and redoxrelated molecules in the placenta in preeclampsia and fetal growth restriction. Virchows Archiv. 2004;444:49-55.

8. Myatt L, Kossenjans W, Sahay R, Eis A, Brockman D. Oxidative stress causes vascular dysfunction in the placenta. The Journal of Maternal-Fetal Medicine. 2015;9:79-82.

9. Jiang C, Jiang L, Li Q, Liu X, Zhang T, Dong L, et al. Acrolein induces NLRP3 inflammasome-mediated pyroptosis and suppresses migration via ROS-dependent autophagy in vascular endothelial cells. Toxicology. 2018;410:26-40.

10. Hu C, Yang Y, Li J, Wang H, Tan C. Maternal Diet-Induced Obesity Compromises Oxidative Stress Status and Angiogenesis in the Porcine Placenta by Upregulating Nox2 Expression. Oxidative Medicine and Cellular Longevity. 2019;2019:1-13.

11. Luo Z, Xu X, Sho T, Luo W, Zhang J, Xu W, et al. Effects of n-acetyl-cysteine supplementation in late gestational diet on maternal-placental redox status, placental NLRP3 inflammasome, and fecal microbiota in sows1. J Anim Sci. 2019;97:1757-71.

12. Mou D, Wang J, Liu H, Chen Y, Che L, Fang Z, et al. Maternal methyl donor supplementation during gestation counteracts bisphenol A-induced oxidative stress in sows and offspring. Nutrition (Burbank, Los Angeles County, Calif). 2018;45:76-84.

13. Liu H, Bai M, Tan B, Xu K, Yu R, Huang R, et al. Influence of supplemented coated-cysteamine on morphology, apoptosis and oxidative stress status of gastrointestinal tract. BMC Vet Res. 2019;15:328.

14. Paul BD, Snyder SH. Therapeutic Applications of Cysteamine and Cystamine in Neurodegenerative and Neuropsychiatric Diseases. Frontiers in Neurology. 2019;10:1315. 
15. Okamura DM, Bahrami NM, Ren S, Pasichnyk K, Williams JM, Gangoiti JA, et al. Cysteamine modulates oxidative stress and blocks myofibroblast activity in CKD. Journal of the American Society of Nephrology. 2014;25:43-54.

16. Paintlia MK, Paintlia AS, Singh AK, Singh I. Attenuation of lipopolysaccharide-induced inflammatory response and phospholipids metabolism at the feto-maternal interface by $\mathrm{N}$-acetyl cysteine. Pediatric Research. 2008;64:334-9.

17. Wilson ME, Biensen NJ, Ford SP. Novel insight into the control of litter size in pigs, using placental efficiency as a selection tool. J Anim Sci. 1999;77:1654-8.

18. Huang S, Wei J, Yu H, Hao X, Zuo J, Tan C, et al. Effects of Dietary Fiber Sources during Gestation on Stress Status, Abnormal Behaviors and Reproductive Performance of Sows. Animals (Basel). 2020;10.

19. Hu C, Yang Y, Deng M, Yang L, Wu G. Placentae for Low Birth Weight Piglets Are Vulnerable to Oxidative Stress, Mitochondrial Dysfunction, and Impaired Angiogenesis. Oxidative Medicine and Cellular Longevity. 2020;2020:1-12.

20. Yu N, Wu JL, Xiao J, Fan L, Chen SH, Li W. HIF-1a regulates angiogenesis via Notch1/STAT3/ETBR pathway in trophoblastic cells. Cell cycle (Georgetown, Tex). 2019;18:3502-12.

21. Poston L, Raijmakers MTM. Trophoblast oxidative stress, antioxidants and pregnancy outcome--a review. Placenta. 2004;25:S72-S8.

22. Gu XL, Li H, Song ZH, Ding YN, He X, Fan ZY. Effects of isomaltooligosaccharide and Bacillus supplementation on sow performance, serum metabolites, and serum and placental oxidative status. Anim Reprod Sci. 2019;207:52-60.

23. Kraeling RR, Webel SK. Current strategies for reproductive management of gilts and sows in North America. J Anim Sci Biotechnol. 2015;6:3.

24. Cai S, Zhu J, Zeng X, Ye Q, Ye C, Mao X, et al. Maternal N-Carbamylglutamate Supply during Early Pregnancy Enhanced Pregnancy Outcomes in Sows through Modulations of Targeted Genes and Metabolism Pathways. Journal of agricultural and food chemistry. 2018;66:5845-52.

25. Bazer FW, Song G, Kim J, Dunlap KA, Satterfield MC, Johnson GA, et al. Uterine biology in pigs and sheep. J Anim Sci Biotechnol. 2012;3:23.

26. Zhao S, Zhong S, Wang F, Wang H, Xu D, Li G. Microcystin-LR exposure decreased the fetal weight of mice by disturbance of placental development and ROS-mediated endoplasmic reticulum stress in the placenta. Environmental pollution (Barking, Essex : 1987). 2020;256:113362.

27. Leung DN, Smith SC, To KF, Sahota DS, Baker PN. Increased placental apoptosis in pregnancies complicated by preeclampsia. American Journal of Obstetrics \& Gynecology. 2001;184:1249-50.

28. Smith SC, Baker PN, Symonds EM. Increased placental apoptosis in intrauterine growth restriction. American Journal of Obstetrics \& Gynecology. 1997;177:1395-401.

29. Wang J, Feng C, Liu T, Shi M, Wu G, Bazer FW. Physiological alterations associated with intrauterine growth restriction in fetal pigs: Causes and insights for nutritional optimization. Molecular reproduction and development. 2017;84:897-904. 
30. Myatt L, Cui X. Oxidative stress in the placenta. Histochemistry and cell biology. 2004;122:369-82.

31. Yang Y, Hu CJ, Zhao X, Xiao K, Deng M, Zhang L, et al. Dietary energy sources during late gestation and lactation of sows: effects on performance, glucolipid metabolism, oxidative status of sows, and their offspring1. J Anim Sci. 2019;97:4608-18.

32. Zhang B, Wang C, Yang W, Zhang H, Meng Q, Shi B, et al. Transcriptome analysis of the effect of pyrroloquinoline quinone disodium (PQQ $\mathrm{Na}(2)$ ) on reproductive performance in sows during gestation and lactation. J Anim Sci Biotechnol. 2019;10:62.

33. Jonker FH. Fetal death: comparative aspects in large domestic animals. Animal Reproduction Science. 2004;s 82-83:415-30.

34. Takashi, Ozaki, Masaki, Kaibori, Kosuke, Matsui, et al. Effect of Thiol-Containing Molecule Cysteamine on the Induction of Inducible Nitric Oxide Synthase in Hepatocytes. Journal of Parenteral and Enteral Nutrition. 2017;31:366-71.

35. Besouw M, Masereeuw R, Lambert VDH, Levtchenko E. Cysteamine: an old drug with new potential. Drug Discovery Today. 2013;18:785-92.

36. Trocino RA, Akazawa S, Ishibashi M, Matsumoto K, Matsuo H, Yamamoto H, et al. Significance of glutathione depletion and oxidative stress in early embryogenesis in glucose-induced rat embryo culture. Diabetes. 1995;44:992-8.

37. Yin J, Ren W, Yang G, Duan J, Huang X, Fang R, et al. L-Cysteine metabolism and its nutritional implications. Molecular nutrition \& food research. 2016;60:134-46.

38. Herrera EA, Cifuentes-Zúñiga F, Figueroa E, Villanueva C, Hernández C, Alegría R, et al. NAcetylcysteine, a glutathione precursor, reverts vascular dysfunction and endothelial epigenetic programming in intrauterine growth restricted guinea pigs. The Journal of physiology. 2017;595:1077-92.

39. Jeitner TM, Lawrence DA. Mechanisms for the cytotoxicity of cysteamine. Toxicological sciences : an official journal of the Society of Toxicology. 2001;63:57-64.

40. Carter AM. Evolution of placental function in mammals: the molecular basis of gas and nutrient transfer, hormone secretion, and immune responses. Physiological reviews. 2012;92:1543-76.

41. Pereira RD, De LNE, Wang RC, Yazdi FT, Holloway AC, Sandeep R. Angiogenesis in the Placenta: The Role of Reactive Oxygen Species Signaling. Biomed Research International. 2015;2015:814543.

42. Wilson ME, Biensen NJ, Youngs CR, Ford SP. Development of Meishan and Yorkshire littermate conceptuses in either a Meishan or Yorkshire uterine environment to day 90 of gestation and to term. Biol Reprod. 1998;58:905-10.

43. Reynolds LP, Redmer DA. Angiogenesis in the Placenta1. Biology of Reproduction. 2001;64:1033-40.

44. Touyz RM, Briones AM, Sedeek M, Burger D, Montezano AC. NOX isoforms and reactive oxygen species in vascular health. Molecular interventions. 2011;11:27-35.

45. Khomenko T, Deng X, Ahluwalia A, Tarnawski A, Patel KN, Sandor Z, et al. STAT3 and importins are novel mediators of early molecular and cellular responses in experimental duodenal ulceration. 


\section{Figures}

A

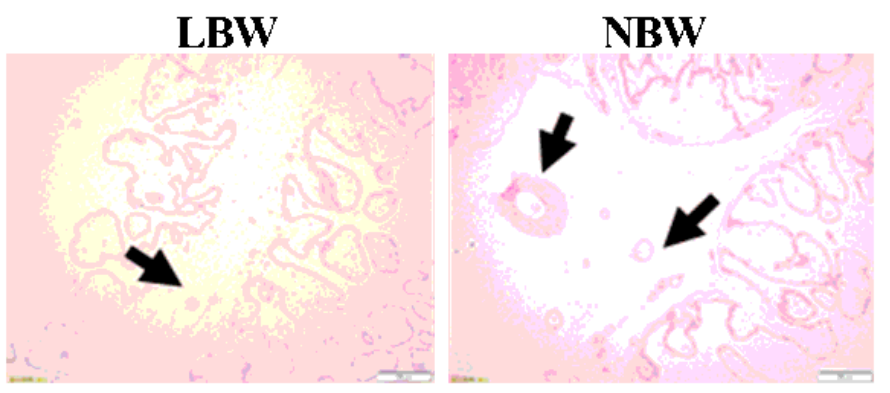

C

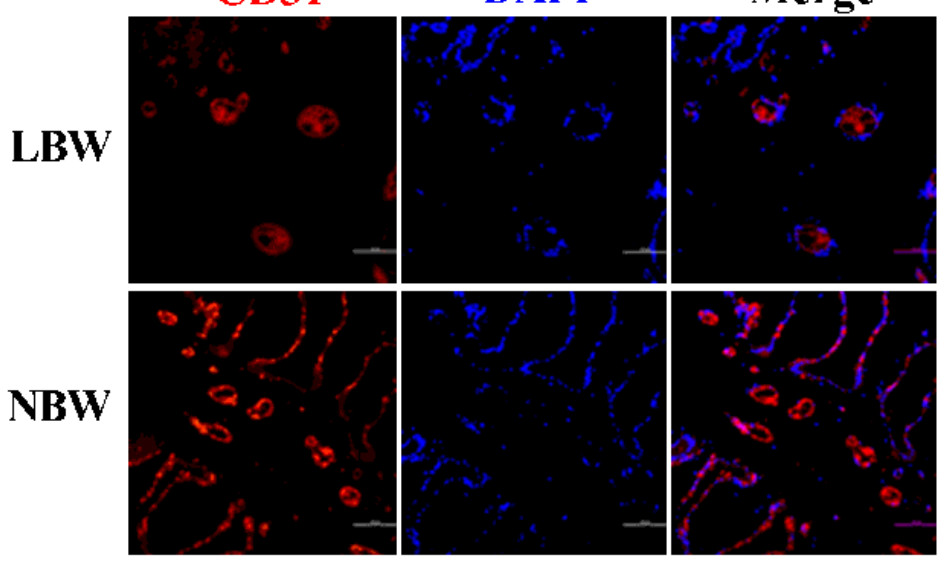

$\mathbf{E}$

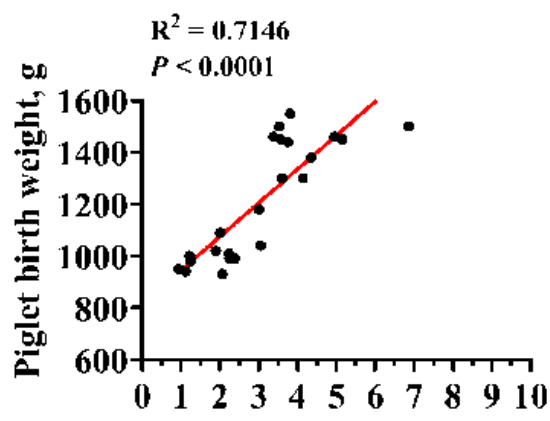

Placental vascular density, $\%$
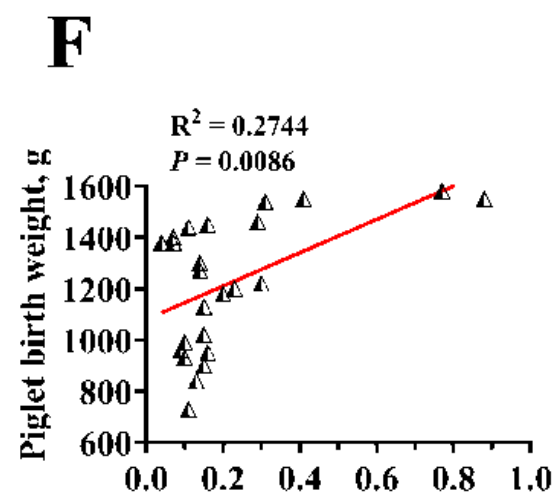

Placental T-AOC,mmol/gprot
B

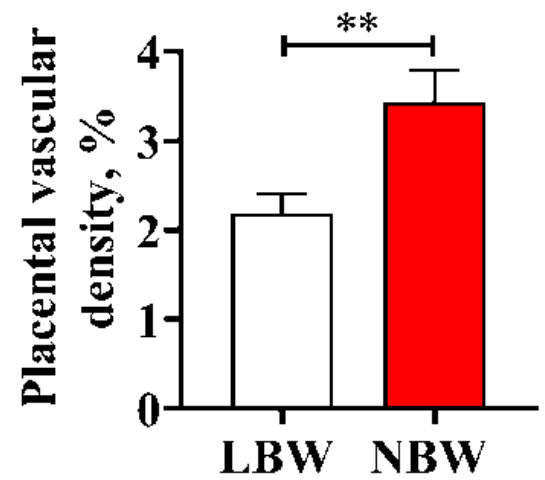

D

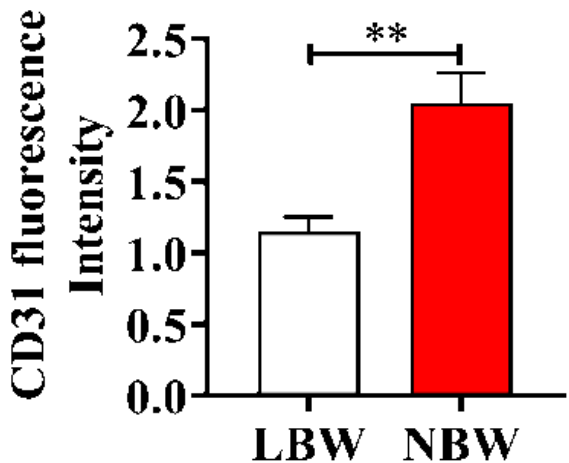

G

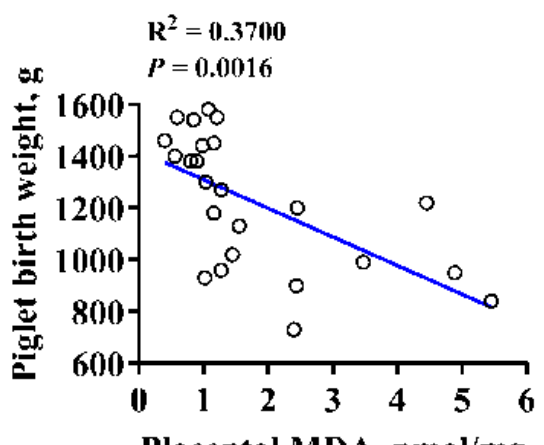

Placental MDA, nmol/mg

\section{Figure 1}

The vessel density distribution in the LBW and NBW placentae. A, B The hematoxylin and eosin method was used to examine blood vessel density in the LBW and NBW placental tissues, and the black arrows 
indicate placental blood vessels ( $b a r=100 \mu m, n=24)$. C, D CD31 immunofluorescence staining in the LBW and NBW placentae. E Correlation between placental blood vessel density and piglet birth weight ( $\mathrm{n}$ = 24). F Correlation between placental total antioxidant capacity (T-AOC) and piglet birth weight $(n=24)$. G Correlation between placental malondialdehyde (MDA) and piglet birth weight $(n=24)$. LBW and NBW indicate piglets with a low birth weight (LBW) (less than $1.0 \mathrm{~kg}$ ) and normal birth weight (NBW) (1.4-1.6 $\mathrm{kg})$, respectively. Data are presented as mean \pm SEM. Differences between groups were considered statistically significant at * $P<0.05, * \star P<0.01, \star \star \star P<0.001$.
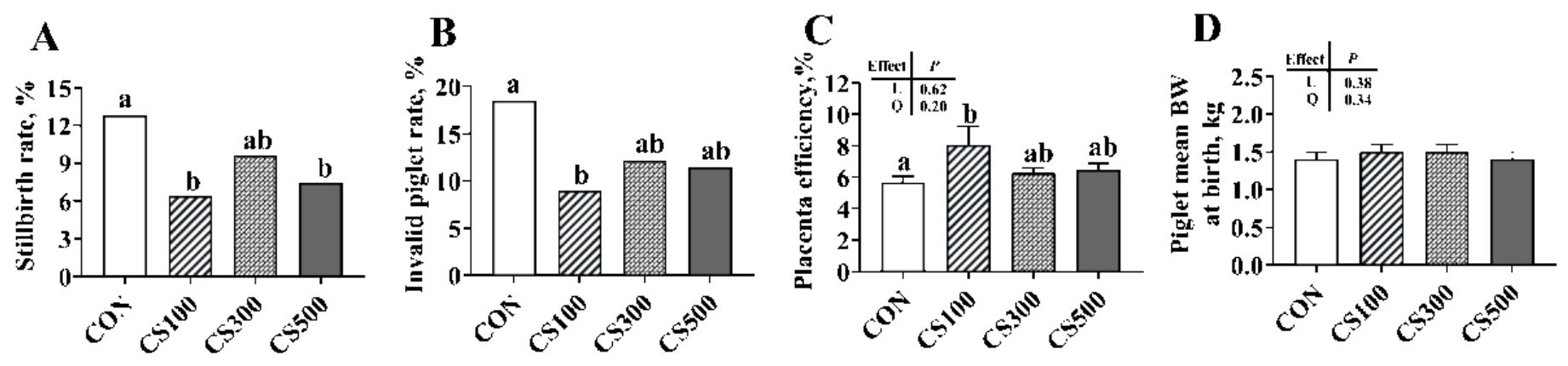

\section{Low yield sows}

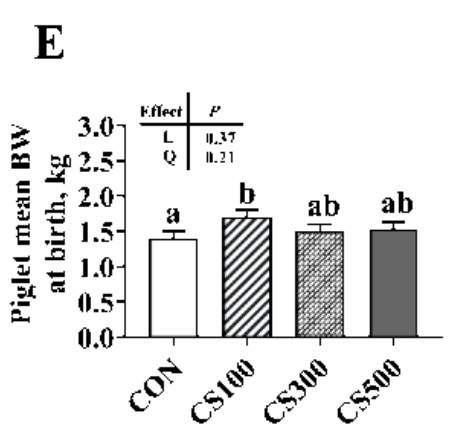

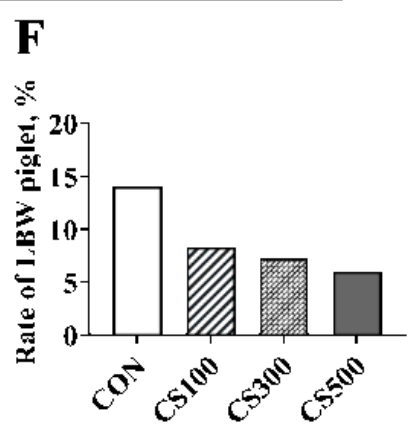

\section{High yield sows}

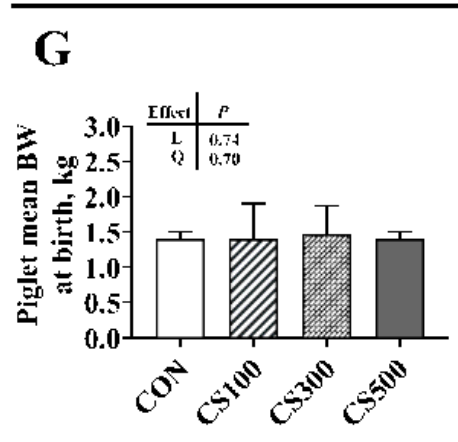

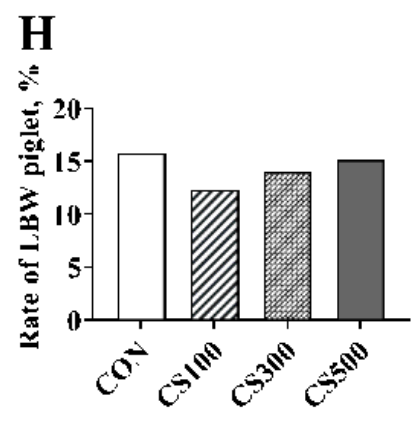

Figure 2

Characteristics of sows and piglets when supplemented with different amounts of cysteamine (CS). A, B Stillbirth and invalid piglet rates were analyzed using the Chi-square test; $C$ Placental efficiency = piglet weight (g)/placental weight (g); D Piglet birth weight, with the number of sows being 21, 20, 19 and 19 in CON, CS100, CS300 and CS500, respectively (a-d); E, F Piglet birth weight and low birth weight (LBW, < $1.0 \mathrm{~kg}$ ) in low yield sows (i.e., the number of piglets born alive is less than 15), with the number of sows being 11, 6, 8 and 9 in CON, CS100, CS300 and CS500, respectively (e-f); G, H Piglet birth weight and normal birth weight (NBW) in high yield sows (i.e., the number of piglets born alive is more than 15), with the number of sows being 10,14, 11 and 10 in CON, CS100, CS300 and CS500, respectively (g-h); CON, basal diet group; CS100/300/500, basal diet supplemented with 100,300, or $500 \mathrm{mg} / \mathrm{kg}$ of CS; Except for rates of stillbirth, invalid piglets, and LBW piglets, all values are expressed as means $\pm S E M$. Linear $(L)$ and quadratic (Q) effects of inclusion amounts of CS were contrasted. Different lowercase letters represent significant difference at $\mathrm{P}<0.05$. 


\section{SOW}
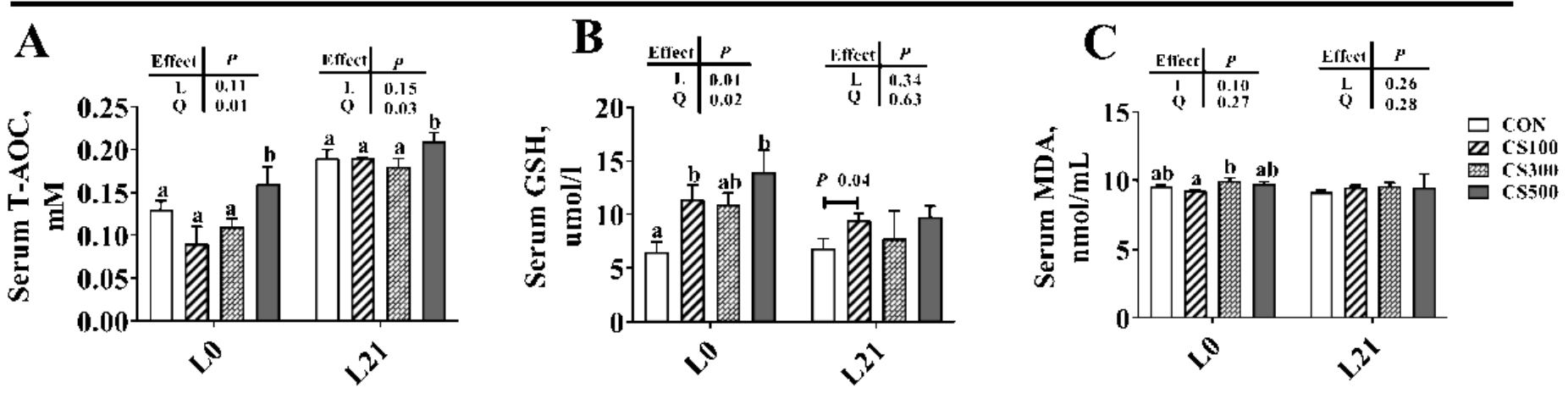

\section{colostrum}
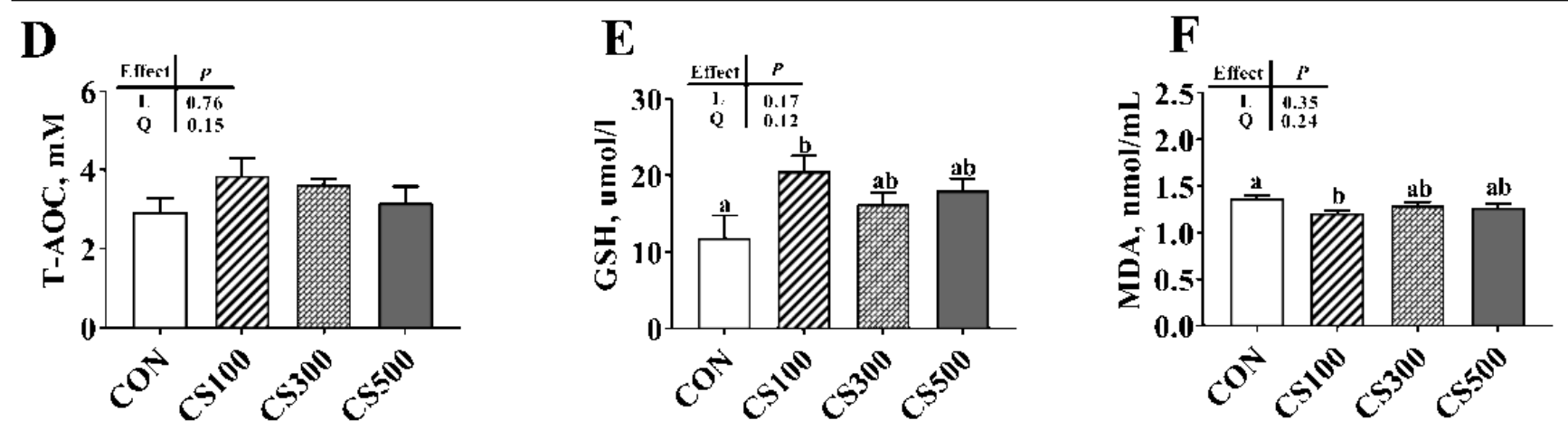

NBW
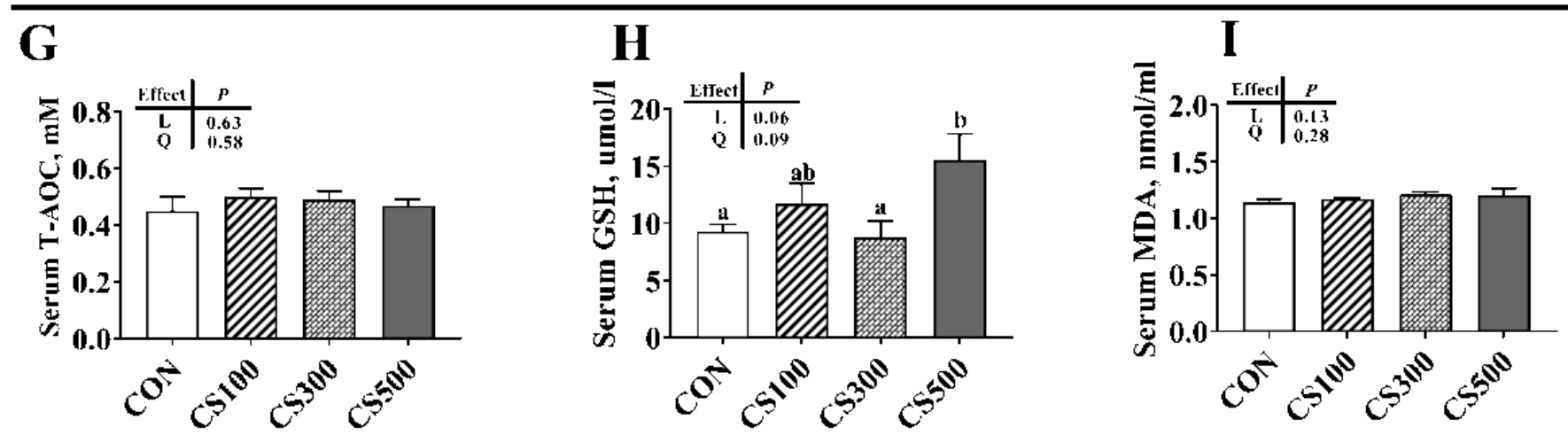

Figure 3

Oxidative stress levels of sows and piglets. A, D, and G T-AOC, total antioxidant capacity; LO, at farrowing; L21, at weaning. B, E, and H GSH, glutathione; C, F, and I MDA, malondialdehyde. CON, basal diet group; CS100/300/500, basal diet supplemented with 100,300 , or $500 \mathrm{mg} / \mathrm{kg}$ of Cysteamine; LBW and NBW indicate piglets with a low birth weight (LBW) (less than $1.0 \mathrm{~kg}$ ) and normal birth weight (NBW) (1.4-1.6 $\mathrm{kg})$, respectively; Data are presented as mean \pm SEM $(n=6-8)$. Linear $(\mathrm{L})$ and quadratic $(\mathrm{Q})$ effects of inclusion amounts of CS were contrasted. Different lowercase letters represent significant difference at $P$ $<0.05$. 

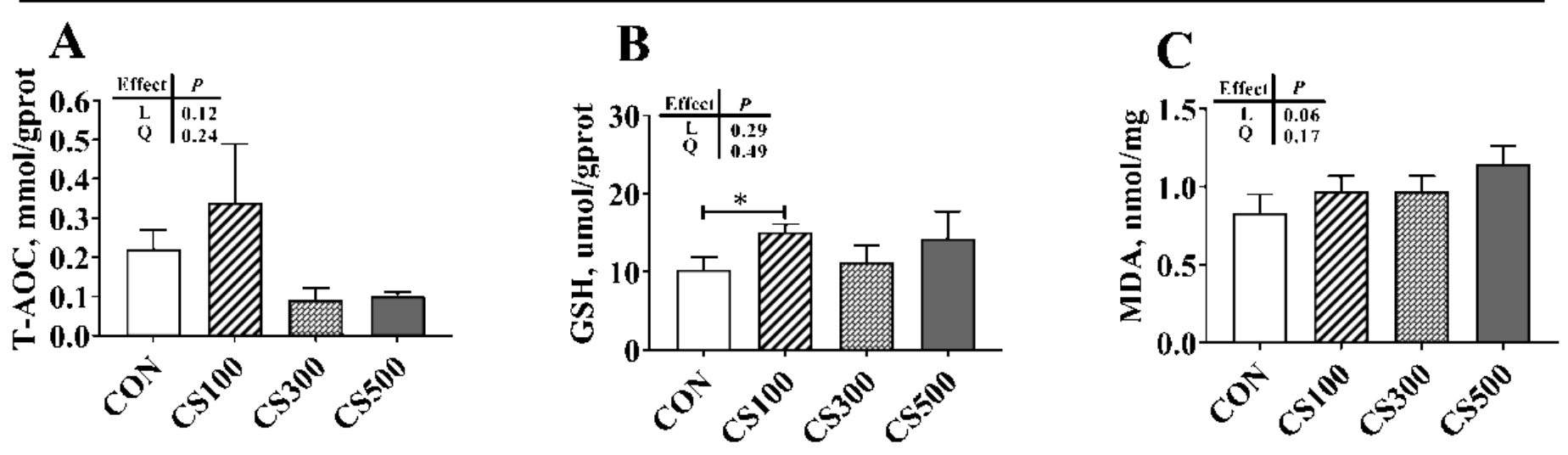

\section{LBW placenta}
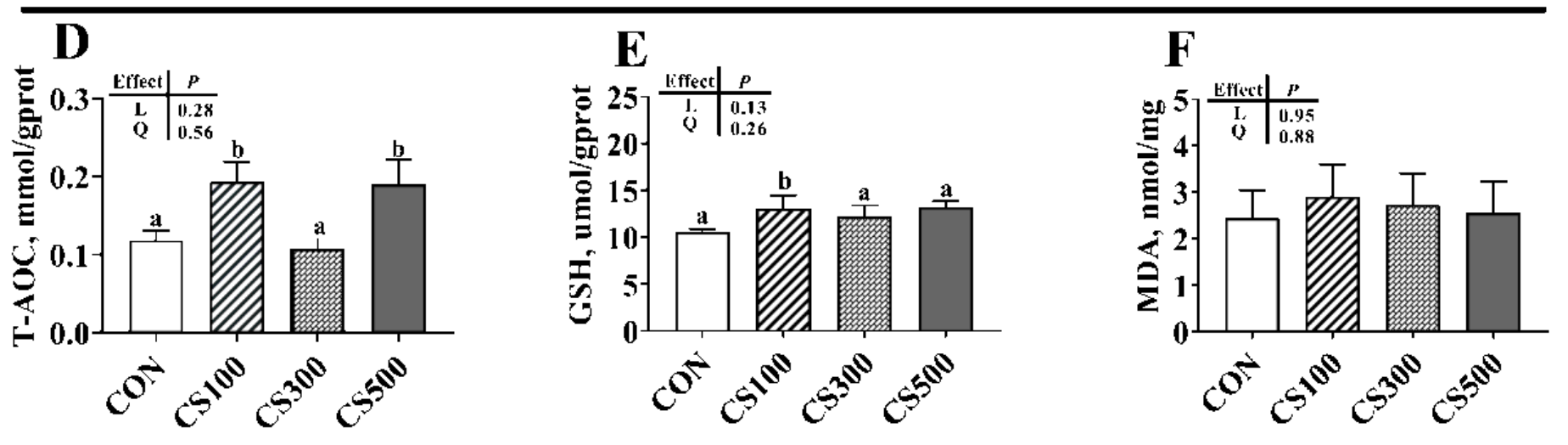

Figure 4

Placental oxidative stress levels. The levels of total antioxidant capacity (T-AOC) (A, D), glutathione (GSH) $(B, E)$, and malondialdehyde $(M D A)(C, F)$ were normalized to the placental total protein. LBW and NBW indicate piglets with a low birth weight (LBW) (less than $1.0 \mathrm{~kg}$ ) and normal birth weight (NBW) (1.4-1.6 $\mathrm{kg}$ ), respectively. CON, basal diet group; CS100/300/500, basal diet supplemented with 100,300, or 500 $\mathrm{mg} / \mathrm{kg}$ of Cysteamine. Data are presented as mean \pm SEM $(n=6)$. Linear $(L)$ and quadratic $(Q)$ effects of inclusion amounts of CS were contrasted. Different lowercase letters represent significant difference at $P$ $<0.05$. 
A

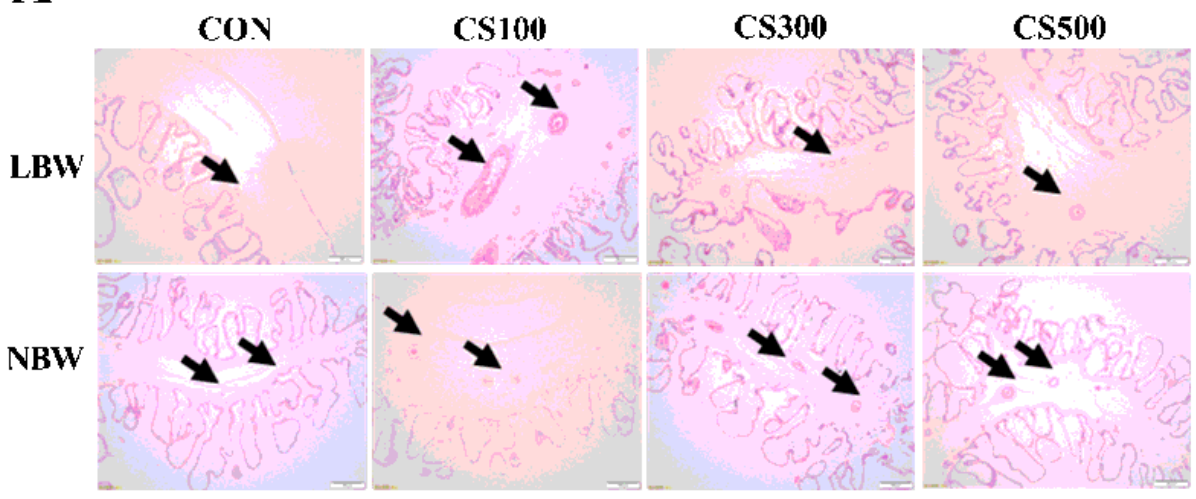

B

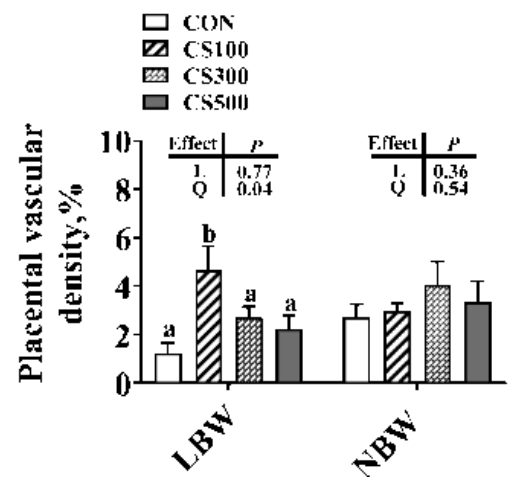

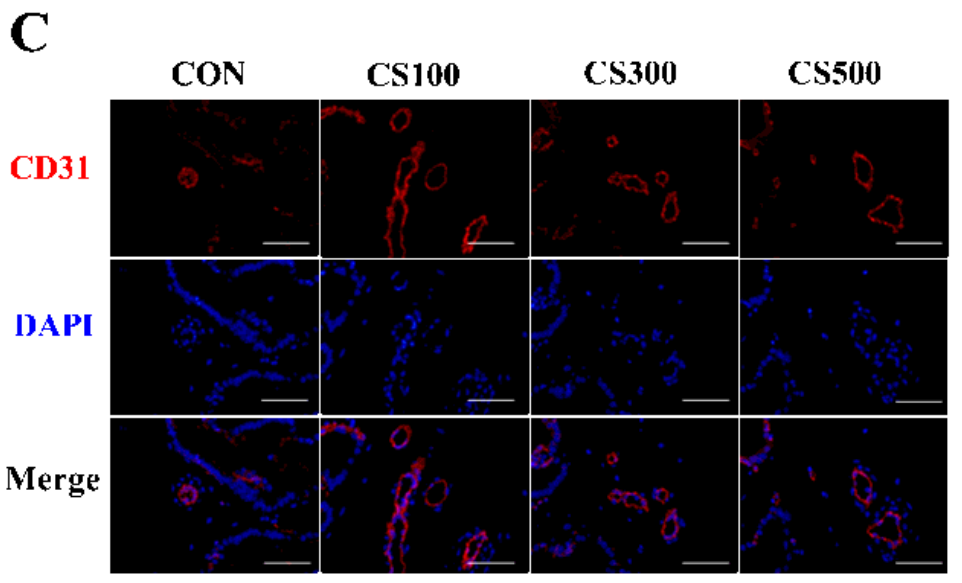

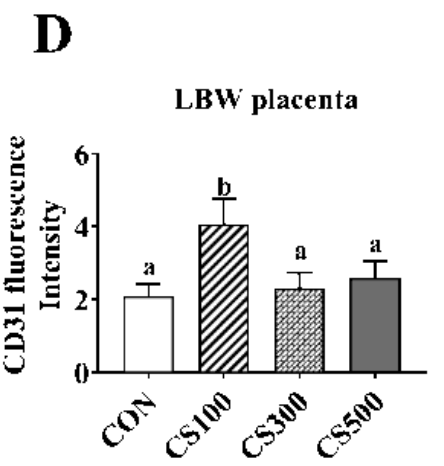

$\mathbf{E}$ L.BW placenta $\checkmark E G F-A$

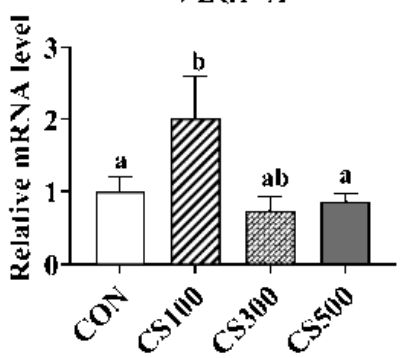

\section{Figure 5}

Effects of maternal cysteamine supplementation on the vascular density of placentae. A, B The hematoxylin and eosin method was used to examine blood vessel density in placental tissues, and the black arrows indicate placental blood vessels (bar $=100 \mu \mathrm{m})$. C, D CD31 immunofluorescence staining in placentae $(\mathrm{bar}=50 \mu \mathrm{m})$. E mRNA relative expression of vascular endothelial growth factor A (VEGF-A). CON, basal diet group; CS100/300/500, basal diet supplemented with 100,300 , or $500 \mathrm{mg} / \mathrm{kg}$ of cysteamine. LBW and NBW indicate piglets with a low birth weight (LBW) (less than $1.0 \mathrm{~kg}$ ) and normal birth weight (NBW) (1.4-1.6 kg), respectively. Data are presented as mean \pm SEM $(n=6)$. Linear $(L)$ and quadratic (Q) effects of inclusion amounts of CS were contrasted. Different lowercase letters represent significant difference at $\mathrm{P}<0.05$. 
A $\quad$ B
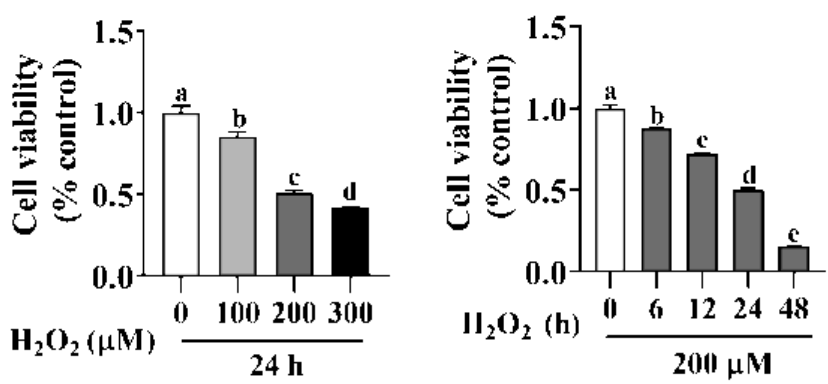

D

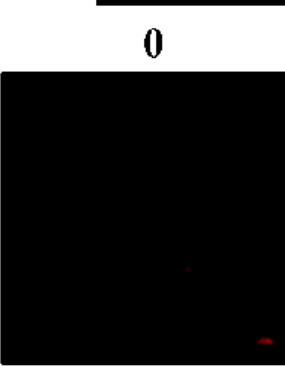

$\mathbf{F}$
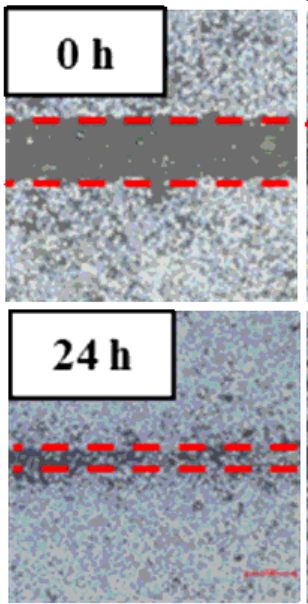

$\mathbf{H}$

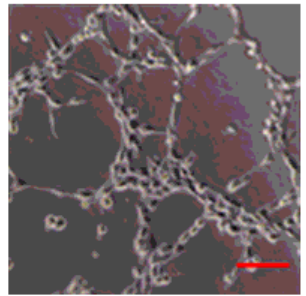

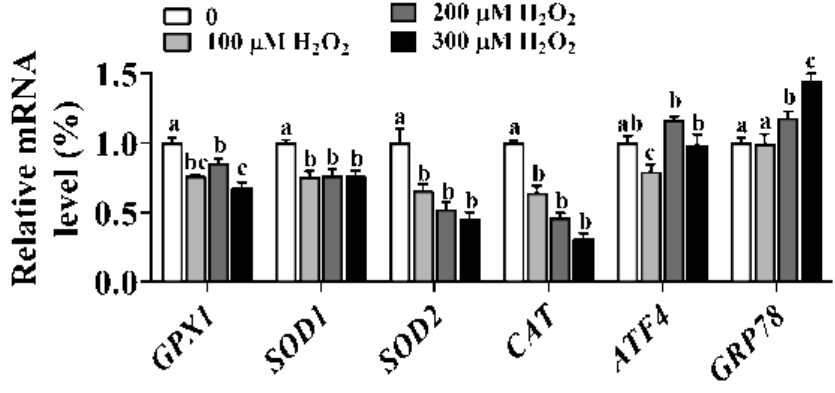

$\mathbf{E}$

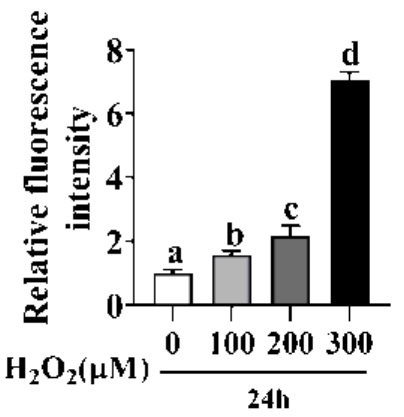

G
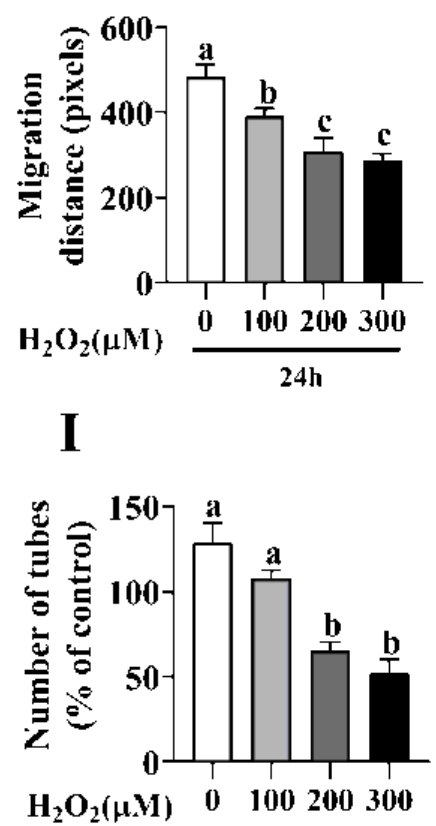

Figure 6

Effects of oxidative stress induced by $\mathrm{H} 2 \mathrm{O} 2$ on the tube formation and migration in porcine vascular endothelial cells (PVECs). A PVECs were treated with various concentrations of $\mathrm{H} 2 \mathrm{O} 2(0,100,200$ or $300 \mu \mathrm{M})$ for $24 \mathrm{~h}(\mathrm{n}=6)$. B PVECs were treated with $200 \mu \mathrm{M} \mathrm{H} 2 \mathrm{O} 2$ for $0,6,12,24$ or $48 \mathrm{~h}$. CCK8 assay was used to measure cell viability $(n=6)$. C The mRNA relative expression of Glutathione peroxidase 1 (GPX1), Cu/Zn superoxide dismutase (SOD1), Mn-superoxide dismutase (SOD2), catalase (CAT), 
activating transcription factor 4 (ATF4), and glucose-regulated protein78 (GPR78). PVECs were treated with various concentrations of $\mathrm{H} 2 \mathrm{O} 2(0,100,200$ or $300 \mu \mathrm{M})$ for $24 \mathrm{~h}(\mathrm{n}=3)$. D, E ROS generation in PVECs treated with various concentrations of $\mathrm{H} 2 \mathrm{O} 2(0,100,200$ or $300 \mu \mathrm{M})$ for $24 \mathrm{~h}(\mathrm{n}=3)$. F, G Scratch healing assay of migratory distance of PVECs treated with various concentrations of $\mathrm{H} 2 \mathrm{O} 2(0,100,200$ or $300 \mu \mathrm{M})$ for $24 \mathrm{~h}(\mathrm{n}=3)$. H, I Representative images of tube formation by PVECs treated with various concentrations of $\mathrm{H} 2 \mathrm{O} 2(0,100,200$ or $300 \mu \mathrm{M})$ for $24 \mathrm{~h}(\mathrm{n}=5$; bar $=50 \mu \mathrm{m})$. Data are presented as mean \pm SEM. Different letters indicate significant differences at $\mathrm{P}<0.05$.

A

B
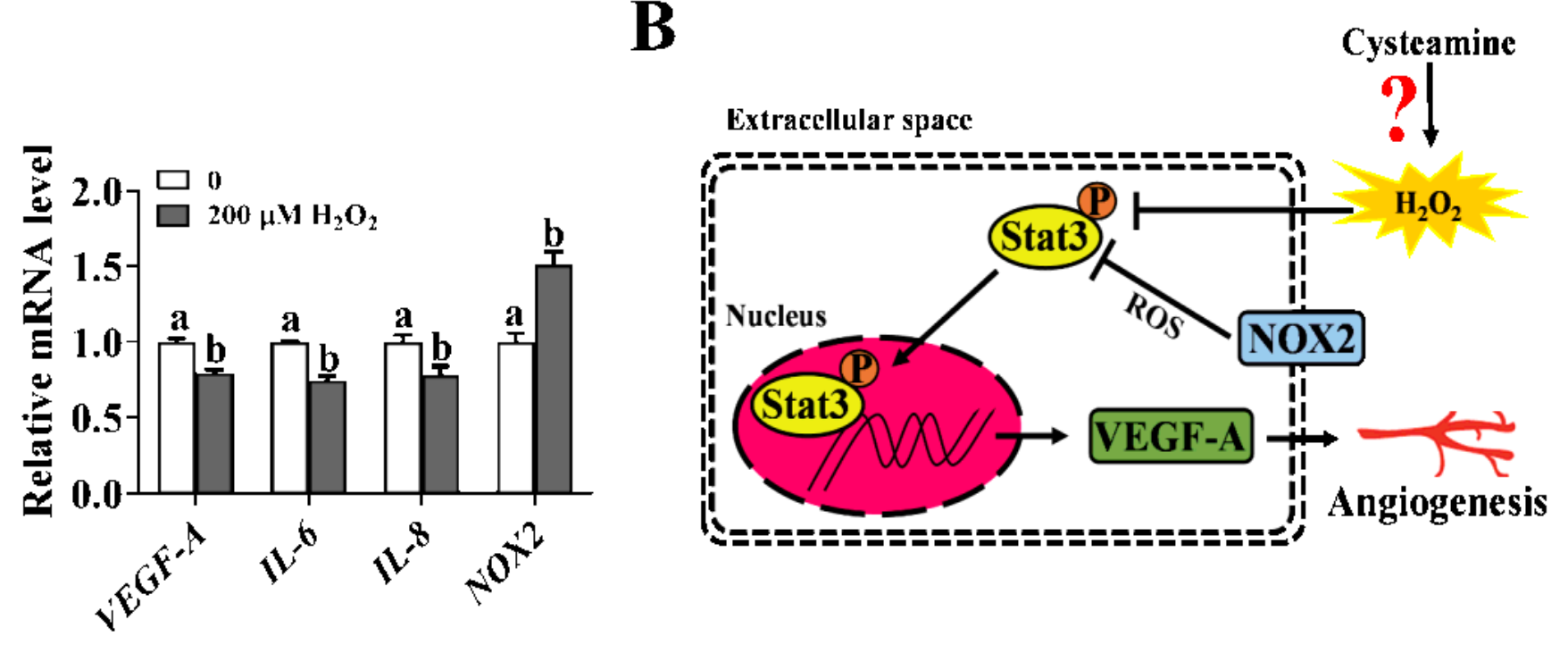

C
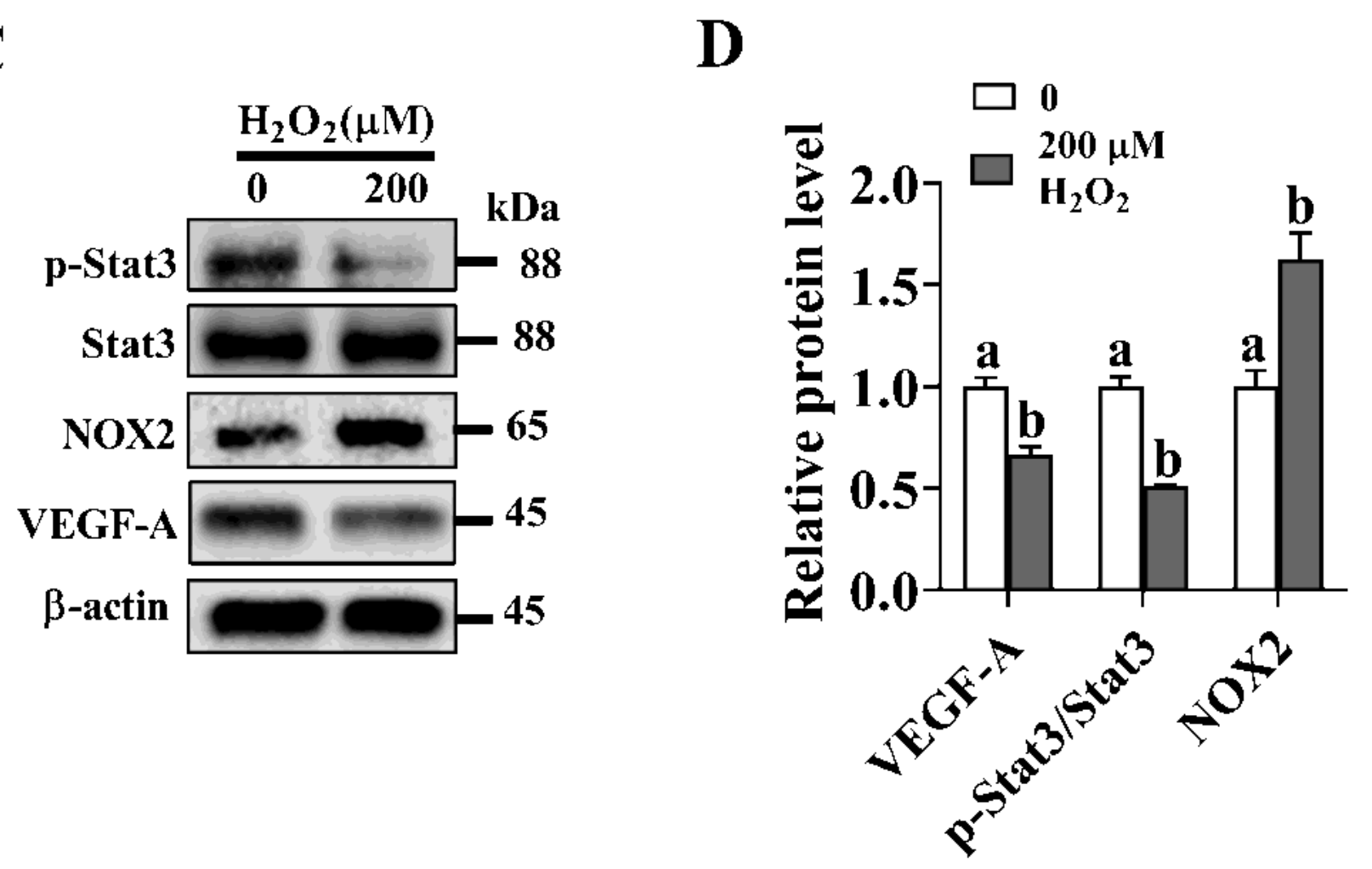
Figure 7

Effects of oxidative stress induced by $\mathrm{H} 2 \mathrm{O} 2$ on the Stat3/VEGF-A pathway in porcine vascular endothelial cells (PVECs). A The mRNA expression of angiogenesis-related factors (vascular endothelial growth factor A, VEGF-A; interleukin-6/8, IL-6/8; NADPH oxidase 2, NOX2). B Schematic for the mechanism of H2O2-induced angiogenesis impairment. C, D Western blotting analysis of the expression of phosphoStat3 (p-Stat3), NOX2, and VEGF-A. Cells were treated with $200 \mu \mathrm{M} \mathrm{H} 2 \mathrm{O} 2$ for $24 \mathrm{~h}$. Data are presented as mean \pm SEM $(n=3)$. Different letters indicate significant differences at $P<0.05$.

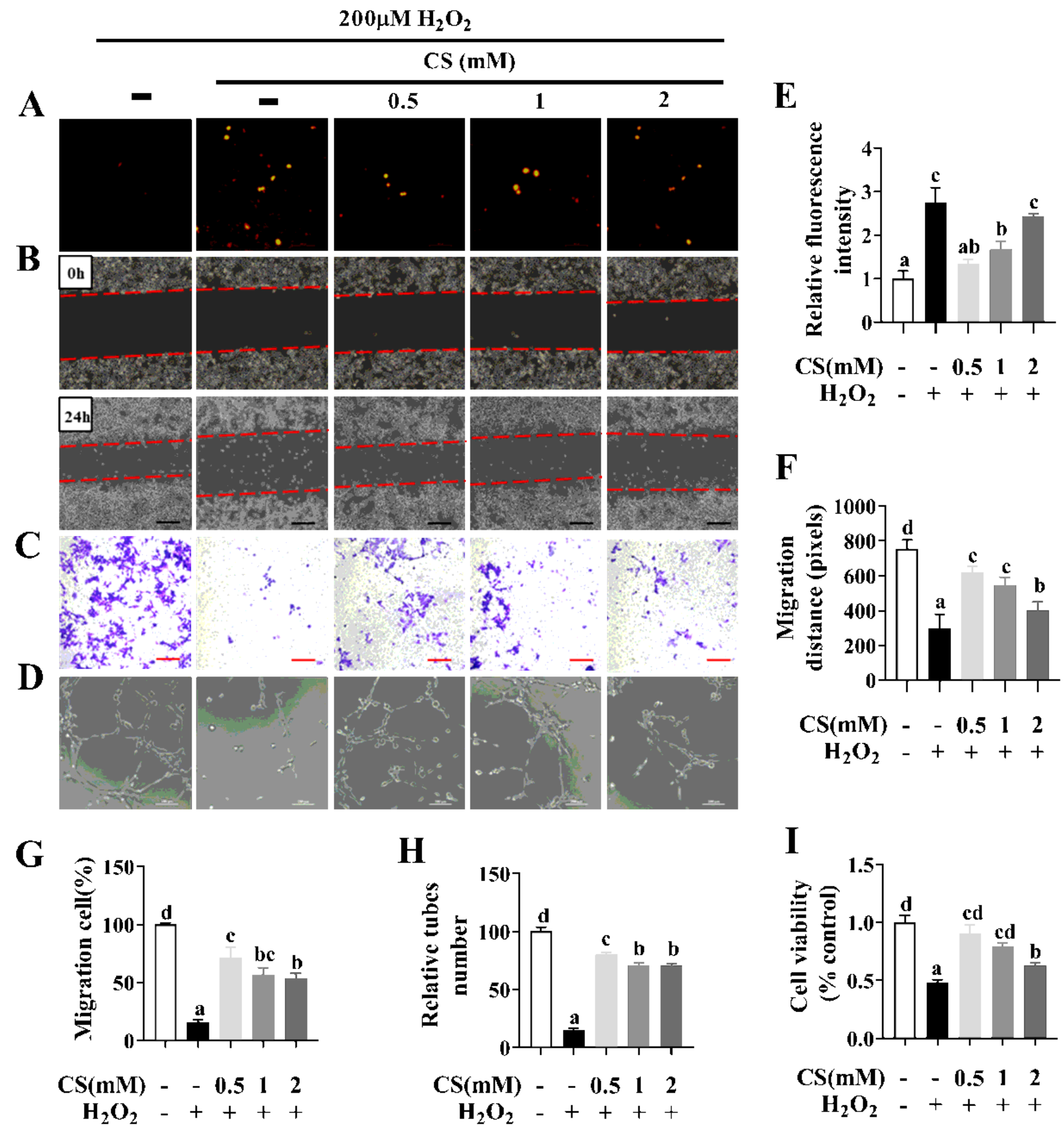




\section{Figure 8}

Cysteamine (CS) pretreatment attenuates the effects of $\mathrm{H} 2 \mathrm{O} 2$ on angiogenesis. A, E The levels of ROS. PVECs were pretreated with various concentrations of CS $(0.5,1$ or $2 \mathrm{mM})$ for $2 \mathrm{~h}$ and then treated with $200 \mu \mathrm{M} \mathrm{H} 2 \mathrm{O} 2$ for $24 \mathrm{~h}(\mathrm{n}=6$; bar $=100 \mu \mathrm{m})$. B, F Scratch healing assay of migratory distance. PVECs were pretreated with various concentrations of CS $(0.5,1$ or $2 \mathrm{mM})$ for $2 \mathrm{~h}$ and then treated with $200 \mu \mathrm{M} \mathrm{H} 2 \mathrm{O} 2$ for $24 \mathrm{~h}(\mathrm{n}=3$; bar $=100 \mu \mathrm{m})$. C, G Trans-well migration assay of the migratory number of PVECs. After different treatments as described above, PVECs were added to the upper chamber of a trans-well. After 48 $h$ incubation, PVECs that invaded through the chamber were quantified $(n=3 ;$ bar $=100 \mu m)$. D, H Representative images of tube formation of PVECs after different treatments as described above $(n=5$; bar $=50 \mu \mathrm{m})$. I CCK8 assay was used to measure cell viability after different treatments as described above $(n=6)$. Data are presented as mean $\pm \operatorname{SEM}(n=3)$. Different letters indicate significant differences at $\mathrm{P}<0.05$. 

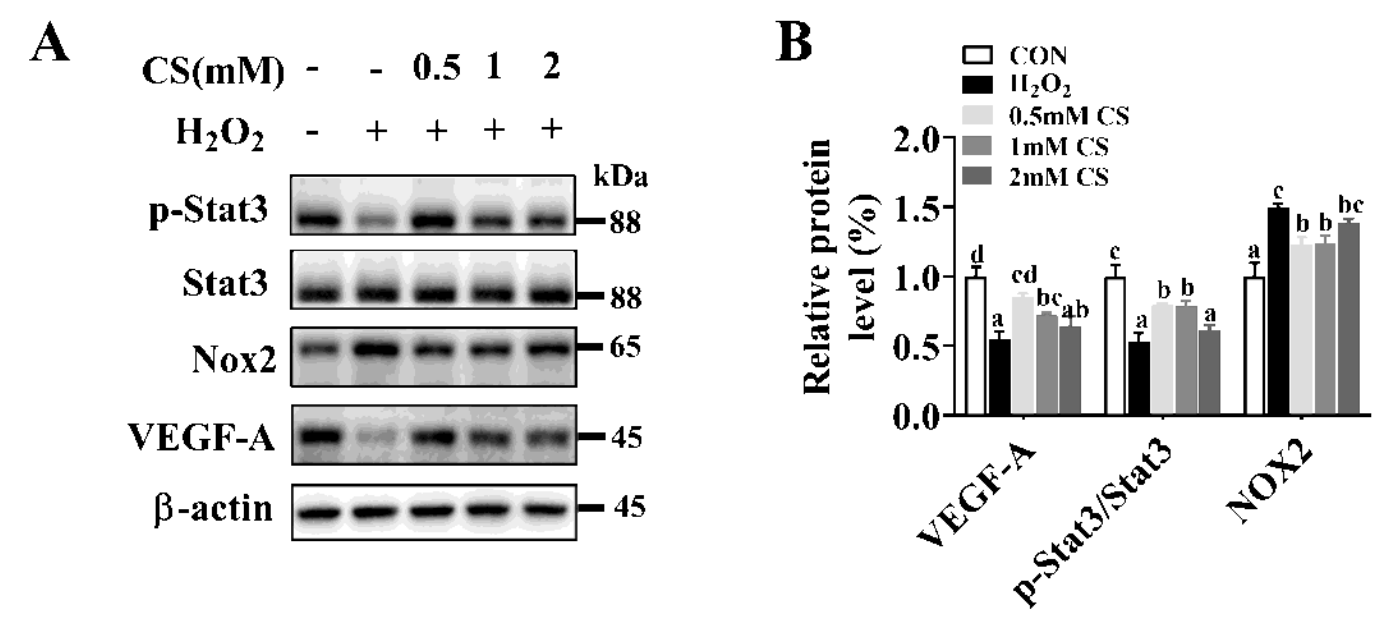

C
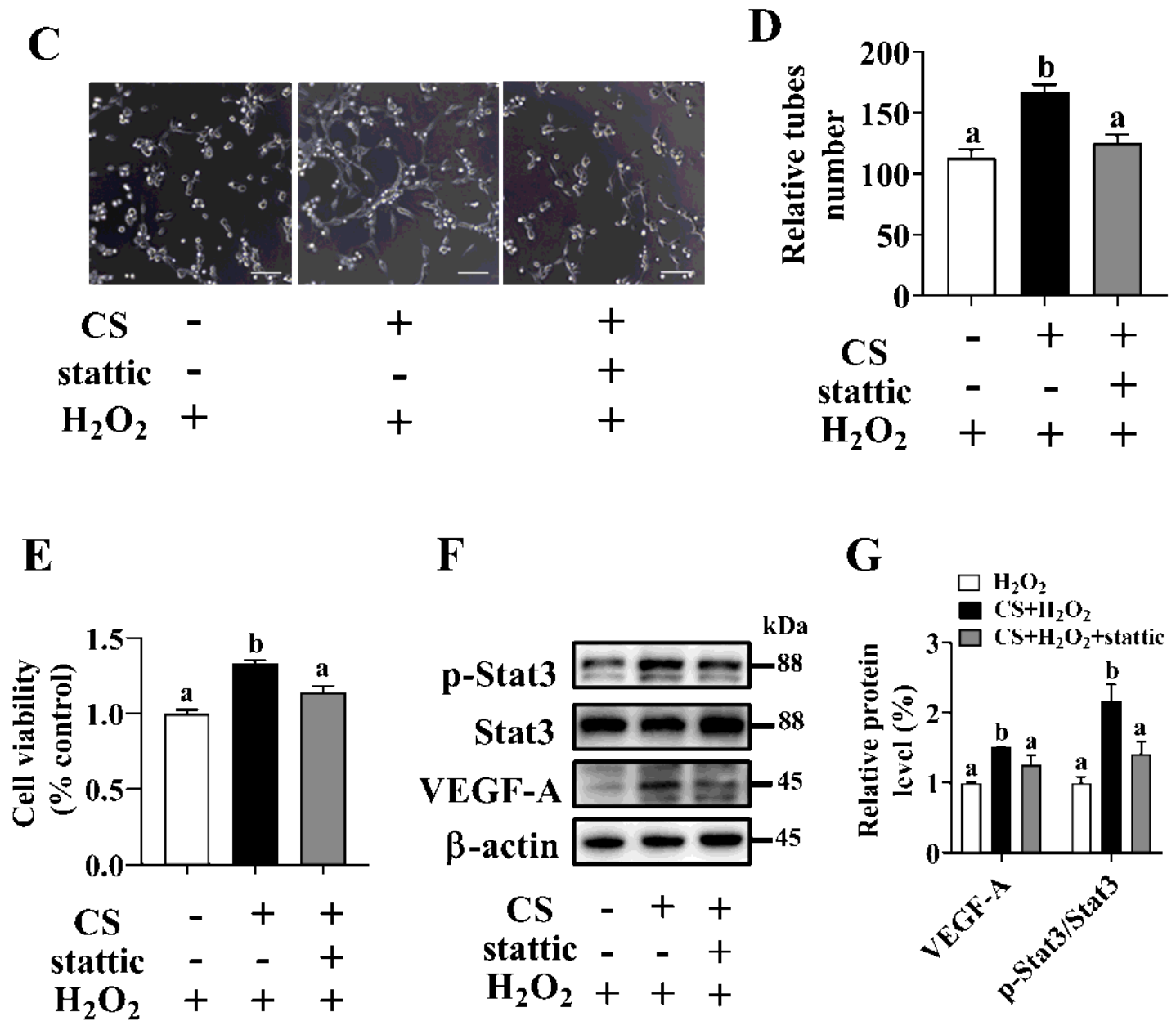

Figure 9

Cysteamine (CS) pretreatment prolongs the phosphorylation of Stat3 in H2O2-treated PVECs. A, B Western blotting analysis of the expression of Phospho-Stat3 (p-Stat3), NADPH oxidase 2 (NOX2), and vascular endothelial growth factor $A$ (VEGF-A). Cells were pretreated with various concentrations of cysteamine $(0.5,1$ or $2 \mathrm{mM} \mathrm{CS})$ for $2 \mathrm{~h}$, and then challenged with $\mathrm{H} 2 \mathrm{O} 2(200 \mu \mathrm{M})$ for $24 \mathrm{~h}(\mathrm{n}=3)$. C, D Representative images of tube formation by PVEC cells, pretreated with CS $(0.5 \mathrm{mM})$ and/or inhibitors of 
Stat3 ( $5 \mu \mathrm{M}$ stattic) for $2 \mathrm{~h}$, and then challenged with $\mathrm{H} 2 \mathrm{O} 2(200 \mu \mathrm{M})$ for $24 \mathrm{~h}(\mathrm{n}=5$; bar $=50 \mu \mathrm{m}) 5$. E CCK8 assay was used to measure cell viability after different treatments as described above $(n=6)$. F, G Western blotting analysis of the expression of Phospho-Stat3 (p-Stat3) and vascular endothelial growth factor $A(V E G F-A)(n=3)$. Data are presented as mean \pm SEM. Different letters indicate significant differences at $\mathrm{P}<0.05$.

\section{Supplementary Files}

This is a list of supplementary files associated with this preprint. Click to download.

- Supplementary.docx 